EPJ manuscript No.

(will be inserted by the editor)

\title{
Standard Model backgrounds to supersymmetry searches
}

\author{
Michelangelo L. Mangano ${ }^{1, a}$ \\ CERN, PH Department, TH Group, 1211 Geneva 23, Switzerland
}

\begin{abstract}
This work presents a review of the Standard Model sources of backgrounds to the search of supersymmetry signals. Depending on the specific model, typical signals may include jets, leptons, and missing transverse energy due to the escaping lightest supersymmetric particle. We focus on the simplest case of multijets and missing energy, since this allows us to expose most of the issues common to other more complex cases. The review is not exhaustive, and is aimed at collecting a series of general comments and observations, to serve as guideline for the process that will lead to a complete experimental determination of size and features of such SM processes.
\end{abstract}

\section{Introduction}

The Standard Model (SM) of fundamental interactions has by now been successfully tested over the past 30 years, validating its dynamics both in the gauge sector, and in the flavour structure, including a compelling confirmation of the source of the observed violation of parity (P) and combined charge and parity (CP) symmetries. The inability of the SM to account for established features of our universe, such as the presence of dark matter, the baryon asymmetry, and neutrino masses, are not considered as flaws of the SM, but as limitations of it, to be overcome by adding new elements, such as new interactions and new fundamental particles. With this perspective, the LHC is not expected to further test the SM, but to probe, and hopefully provide evidence for, the existence of such new phenomena. Our ability to predict what will be observed at the LHC is therefore not limited by fundamental issues related to left-over uncertainties about the SM dynamics, but by the difficulty of mastering the complex strong-interaction dynamics that underlies the description of the final states in proton-proton collisions [1].

Many years of experience at the Tevatron collider, at HERA, and at LEP, have led to an immense improvement of our understanding of this dynamics, and put us today in a solid position to reliably anticipate in quantitative terms the features of LHC final states. LEP, in addition to testing with great accuracy the electroweak interaction sector, has verified at the percent level the predictions of perturbative QCD, from the running of the strong coupling constant, to the description of the perturbative evolution of single quarks and gluons, down to the non-perturbative boundary where strong interactions take over and cause the confinement of partons into hadrons. The description of this transition, relying on the factorization theorem that allows to consistently separate the perturbative and non-perturbative phases, has been validated by the comparison with LEP data, allowing the phenomenological parameters introduced to model hadronization to be determined. The factorization theorem supports the use of these parameters for the description of the hadronization transition in other experimental environments. HERA has made it possible to probe with great accuracy the short-distance

\footnotetext{
a e-mail: michelangelo.mangano@cern.ch
} 
properties of the proton, with the measurement of its partonic content over a broad range of momentum fractions $x$. These inputs, from LEP and from HERA, beautifully merge into the tools that have been developed to describe proton-antiproton collisions at the Tevatron, where the agreement between theoretical predictions and data confirms that the key assumptions of the overall approach are robust. Basic quantities such as the production cross section of $W$ and $Z$ bosons, of jets up to the highest energies, and of top quarks, are predicted theoretically with an accuracy consistent with the known experimental and theoretical systematic uncertainties. This agreement was often reached after several iterations, in which both the data and the theory required improvements and reconsideration. See, for example, the long saga of the bottom-quark cross section [2], or the almost embarrassing — for theorists — case of the production of high transverse momentum $J / \psi \mathrm{s}[3]$.

While the present status encourages us to feel confident about our ability to extrapolate to the LHC, the sometimes tortuous path that led to this success demands caution in assuming by default that we know all that is needed to accurately predict the properties of LHC final states. Furthermore, the huge event rates that will be possible at the LHC, offering greater sensitivity to small deviations, put stronger demands on the precision of the theoretical tools. In this essay I discuss the implications of these considerations, using as a specific example the search for supersymmetry. I shall not provide a systematic discussion of all search strategies, which vary a lot in their details depending on the specific version of the assumed supersymmetry breaking patterns, and of the parameters values. I shall rather focus on the canonical case of the multijet plus missing transverse energy signals, which are well suited to address the rather general background-estimate issues which are the focus of this note. Likewise, I shall not review the state of the art in calculations and Monte Carlo tools (for these, see Refs. [4] and [5], respectively) but will confine myself to the applications of several tools that have recently been developed, and their impact on the expected signals.

For more general considerations on the issue of "discovering new physics at the LHC", I refer the reader to the recent essay in ref. [6].

\section{Signal properties}

To be concrete, I will consider the production and decay of strongly interacting supersymmetric particles, like gluinos and squarks. Chain decays of such particles lead to final states such as those shown in fig. 1. Pair production will therefore typically lead to configuration with several jets, missing transverse energy, and possibly leptons [7]. I shall focus on the case of no-leptons [8]. Final states with leptons $[9,10]$ have backgrounds similar to those studied here, with the addition of possible extra gauge bosons, as well as contributions coming from charm and bottom quarks, which occasionally lead to isolated leptons $[11,12]$. There is a vast literature covering all these facets, and I just refer to the experimental literature for an overview of the state of the art in the current searches at the Tevatron $[13,14]$, and of the prospects for discovery at the LHC [15-21].

The cuts used in the following are the typical analysis cuts chosen by the ATLAS experiment [22-24] to optimize the signal extraction:

$$
\begin{aligned}
& N_{\text {jets }} \geq 4, \text { with } E_{\mathrm{T}}>50 \mathrm{GeV} \text { for all jets, and } E_{\mathrm{T}}>100 \mathrm{GeV} \text { for the leading jet; } \\
& \text { no lepton with } E_{\mathrm{T}}>20 \mathrm{GeV} \\
& E_{T}>\max \left(100 \mathrm{GeV}, 0.2 \times M_{\mathrm{eff}}\right) \\
& M_{\mathrm{eff}}=E_{T}+\sum_{\text {jets }} E_{\mathrm{T}, j} \\
& \text { Transverse sphericity, } S_{T}>0.2
\end{aligned}
$$

A sample result, obtained from the full detector simulation of the backgrounds and of the signal, for a specific point in supersymmetric parameter space, is shown in fig. 2. The signal corresponds to production of squarks and gluinos with a mass of the order of $1 \mathrm{TeV}$, in a typical so-called mSUGRA model, defined by the values of few parameters at the scale of grand unification. The renormalization-group evolution to the electroweak scale leads to the breaking of the gauge 


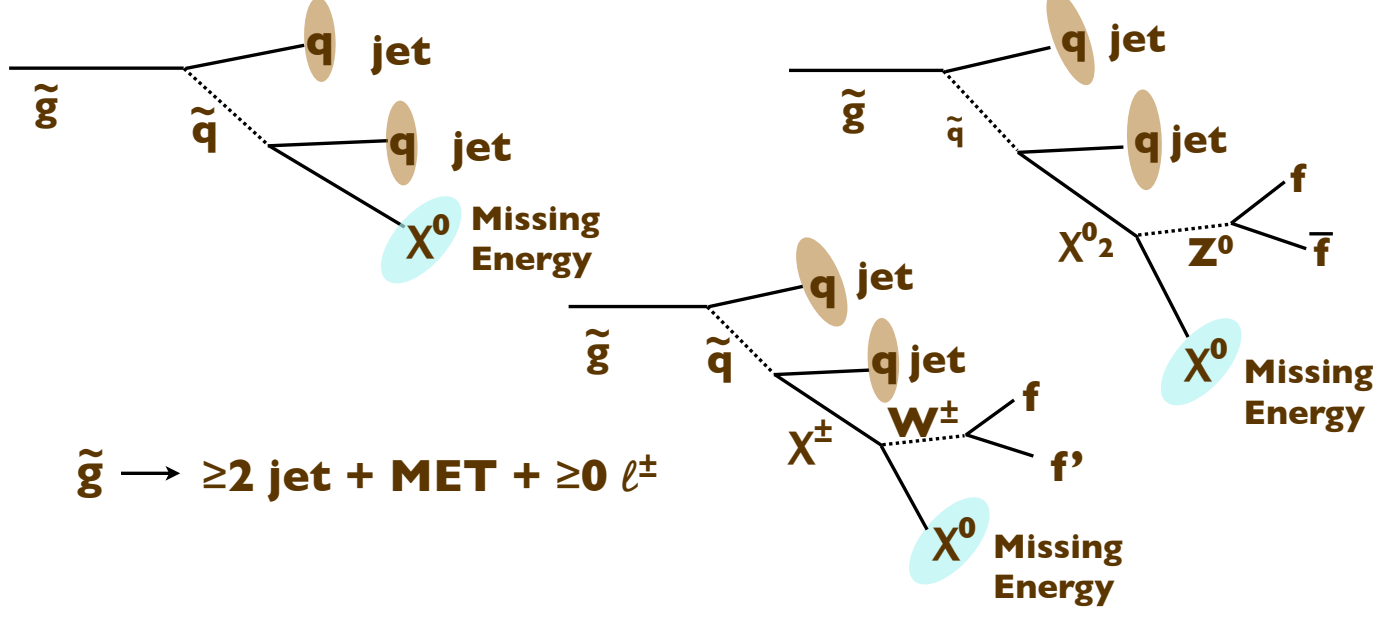

Fig. 1. Possible topologies for the final states of gluino decays.

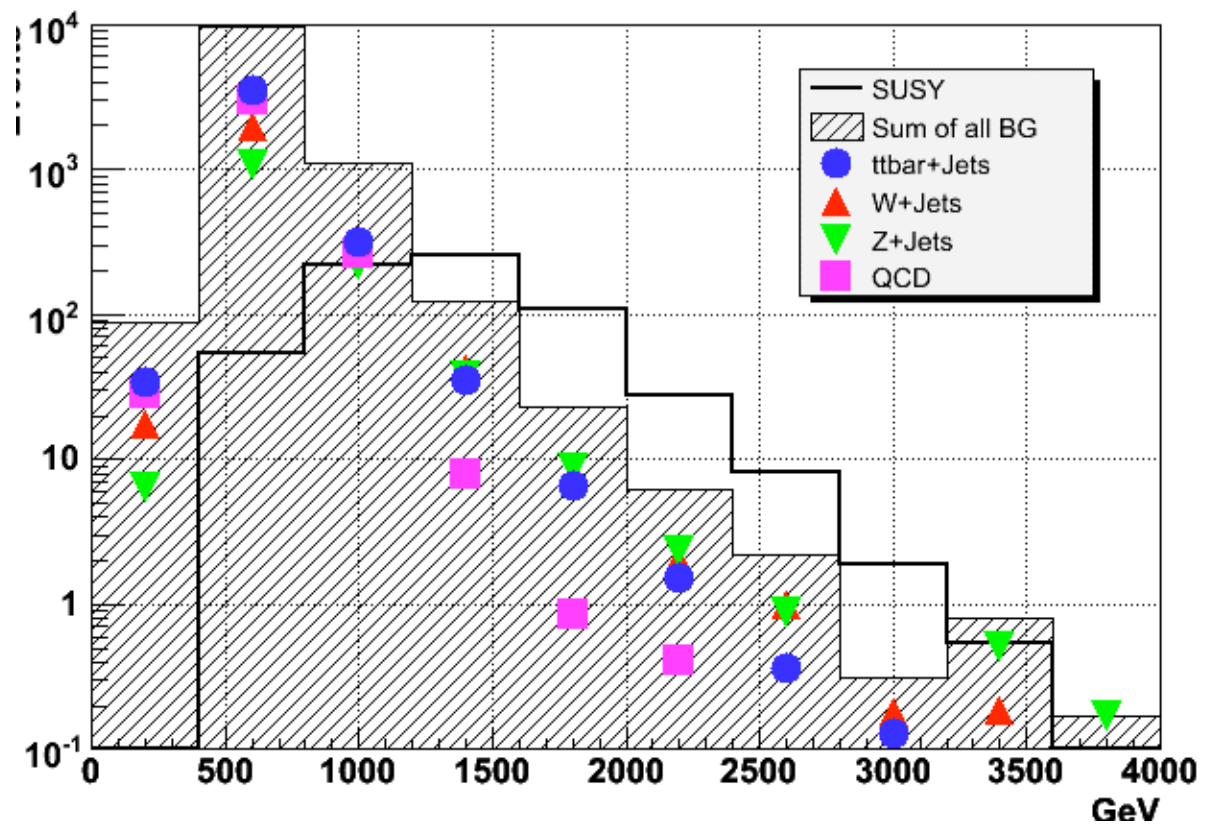

Fig. 2. Example of an expected supersymmetry signal and relative backgrounds in the multijet + missing transverse energy final state $[22]$. 
group $S U(2) \times U(1)$, and defines the entire spectrum of the supersymmetric partners. The parameters are given by the common mass $m_{0}$ ( $400 \mathrm{GeV}$ in our example) of the scalar partners of the Standard Model fermions, by the common mass $m_{1 / 2}(400 \mathrm{GeV})$ of the fermionic partners of the gauge bosons, by the mixing $\tan \beta$ (10) between the expectation values of the two Higgs doublets, by the sign of the mass term $\mu$ of the Higgs fields $(\mu>0)$, and by the parameter $A$ (0) defining the soft-supersymmetry-breaking scalar potential. The resulting gluino mass is $\sim 950 \mathrm{GeV}$, and the mass of the scalar partners of the light quarks is $\sim 930 \mathrm{GeV}$.

The results of fig. 2 are rather general in this class of models: varying the parameters that determine the gluino and squark masses will shift the signal to lower (higher) values of the effective mass $M_{\text {eff }}$, and to higher (lower) rates, depending on whether the resulting sparticle masses are reduced or increased. While the quantitative details of the following analysis will depend on the specific set of chosen parameters, the spirit of the analysis remains the same.

Notice that while the signal has certainly a statistical significance sufficient to claim a deviation from the SM, it is unsettling that its shape is so similar to that of the sum of the backgrounds. The theoretical estimates of these backgrounds have also increased significantly over the last few years, as a result of more accurate tools to describe multijet final states. There is no question, therefore, that unless each of the background components can be separately tested and validated, it will not be possible to draw conclusions from the mere comparison of data against the theory predictions.

I am not saying this because I do not believe in the goodness of our predictions. But because claiming that supersymmetry exists is far too important a conclusion to make it follow from the straight comparison against a Monte Carlo. One should not forget relevant examples from the colliders' history $[25,26]$, such as the misinterpretation in terms of top or supersymmetry of final states recorded by UA1 with jets, $\mathbb{E}_{T}$, and, in the case of top, leptons. Such complex final states were new experimental manifestations of higher-order QCD processes, a field of phenomenology that was just starting being explored quantitatively. It goes to the theorists' credit to have at the time played devil's advocate [27], and to have improved the SM predictions, to the point of proving that those signals were nothing but bread and butter $W$ or $Z$ plus multijet production. But the fact remains that claiming discoveries on the basis of a comparison against a $\mathrm{MC}$ is dangerous.

The lesson for the future is that, more than accurate theoretical calculations, in these cases one primarily needs a strategy for an internal validation of the background estimate. If evidence for some new phenomenon entirely depends on the shape of some distribution, however accurate we think our theoretical inputs are, the conclusion that there is new physics is so important that people will always correctly argue that perhaps there is something weird going on on the theory side, and more compelling evidence has to be given.

In what follows I review the nature of the backgrounds, the status of their theoretical understanding, and the possible approaches to determine them directly from the data.

\section{Background classification}

It is useful to classify backgrounds in three categories: irreducible, reducible, and instrumental.

Irreducible backgrounds are those that, on an event by event basis, cannot be distinguished from the signal, even in presence of a perfect detector. In the case of the supersymmetric signals we are discussing, they emerge from production of $Z$ plus 4 jets, with the $Z$ boson decaying to invisible neutrinos. While the missing mass of the event should reconstruct $M_{Z}$, the tail of the Breit-Wigner distribution can generate events beyond the kinematical range allowed by the neutralino masses. This problem is enhanced by the lack of information on the longitudinal component of the missing-momentum vector.

Reducible backgrounds include processes that share the main features of the signal, but have in addition some extra element that would make them in principle distinguishable from it. The exploitation of these additional elements may be limited, however, either because of the need to maintain a good signal efficiency, or due to a limited experimental sensitivity to the distinguishing elements. Examples of relevance to our case study include: 
- $W+3$ jets, with $W \rightarrow \tau \nu$ and the hadronic decay of the $\tau$ giving rise to the fourth hadronic jet required in the event selection. The $\tau$ can in principle be identified, and rejected, by using both the low multiplicity of the resulting jet, and because, as a result of the $\tau$ lifetime, the charged tracks of the jet originate from a vertex displayed relative to the primary vertex. The efficiency for tagging such $\tau$ decays is however limited, and a residual background will be left. Notice that, due to the presence of the neutrinos from both the $W$ and the $\tau$ decays, in addition to the lack of information on the longitudinal momentum of the neutrinos, the missing mass is also poorly determined, and therefore cannot be used on an event-by-event basis to suppress the background.

$-W+4$ jets, with the $W$ decaying to an undetected lepton and a neutrino. The efficiency to detect, and reject, the leptons, cannot be perfect, since leptons can have low- $p_{\mathrm{T}}$. This is particularly true of $W$ decays to $\tau$, with the $\tau$ itself decaying leptonically.

- $t \bar{t}$ events contain $W$ bosons and jets, and therefore fall automatically under the above categories. The additional presence of $b$ quarks provides a possible handle to reject them, but vetoing on $b$ quarks could be an unwise choice, since the signal itself could have an enhanced fraction of $b$, for example if third-generation squarks were lighter than those of the first two generations. The large rate of $t \bar{t}$ production at the LHC makes this process a potentially large reducible background.

Instrumental backgrounds arise when the characteristic features of the signals are due to the inaccuracy of the detector or of the measurement. The most important example in this category is QCD multijets, namely final states with only jets. The missing transverse momentum is entirely a result of either of the following three effects:

- A mismeasurement of the energy of the individual jets, leading to an overall imbalance of transverse energy

- The incomplete coverage of the calorimeters, which could allow some jets to escape reconstruction, thus leading once again to a momentum imbalance.

- Accidental extra deposits of energy, like cosmic rays hitting part of the detector in time with the recording of the event, backgrounds from protons in the halo of the beam colliding with some detector element upstream of the interaction region, large fluctuations of electronic noise in the calorimeters, etc.

The size of the multijet cross section is huge, much bigger than any possible signal, and therefore even a small contamination of the missing energy measurements can lead to significant backgrounds. As a reference, we show in fig. 3 the $M_{\text {eff }}$ distribution of 4-jet final states from QCD, with jets passing the selection cuts of eq. 2, but without any $\mathbb{E}_{T}$ requirement. To highlight the difficulty of properly removing unwanted sources of $\not_{T}$, we show in fig. 4 the raw spectrum of jet $E_{\mathrm{T}}$ and of $E_{T}$, at D0 and CDF, before and after the removal of events with an identified spurious source of energy deposits. The lower rate of cosmic rays penetrating to the depth of the LHC experiments, the shorter time window allowed by the much higher repetition rate of LHC collisions, and the higher accuracy of the calorimeters, will certainly significantly reduce this problem at the LHC, but the size of the effects certainly poses very difficult experimental challenges.

The prediction for each of these backgrounds, whether their origin is physics or detector effects, as well as possible additional ones, can only partly rely on our a-priori simulation of the physical processes and of the detector performance. The conclusion that a signal for new physics has been identified, to be credible, will have to mostly rely on the data themselves. Each search strategy should therefore contain the definition of control samples and control observables to be used for the direct determination of the backgrounds, e.g. by extrapolating the sidebands of a given distribution, or by validating the $\mathrm{MC}$ simulation tools that will be used to extrapolate our knowledge from the control sample to the signal region. Furthermore, efforts should be made to establish that the extrapolation of the knowledge acquired from the control samples to the signal region is legitimate and reliable. For interesting reviews of how the experiments plan to utilize their data in the process of assessing the size of the backgrounds, see e.g. ref. [23, 24]. 


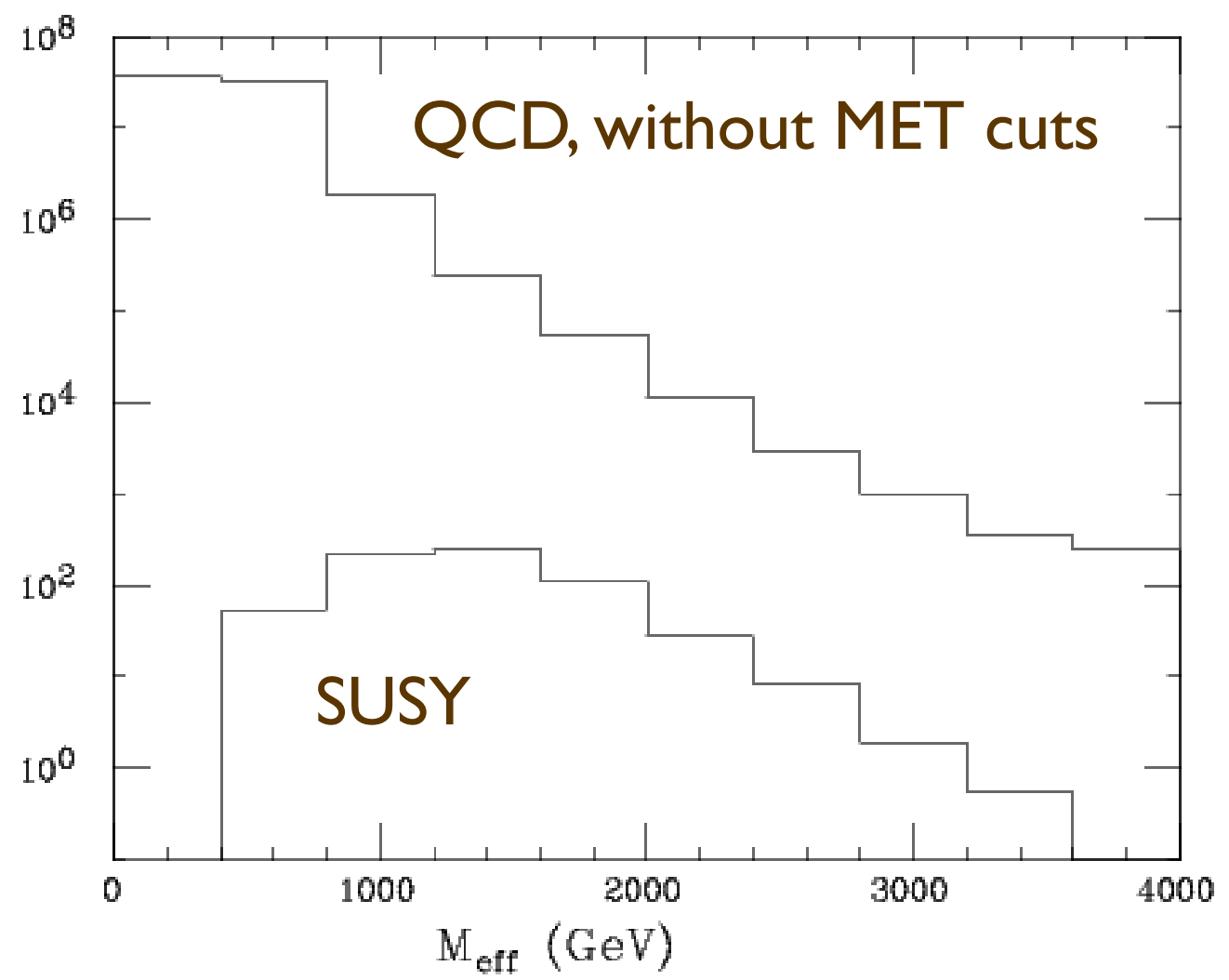

Fig. 3. QCD multijet rates as a function of the effective mass variable $M_{\text {eff }}$, defined in eq. (2), in events without missing transverse energy requirement.
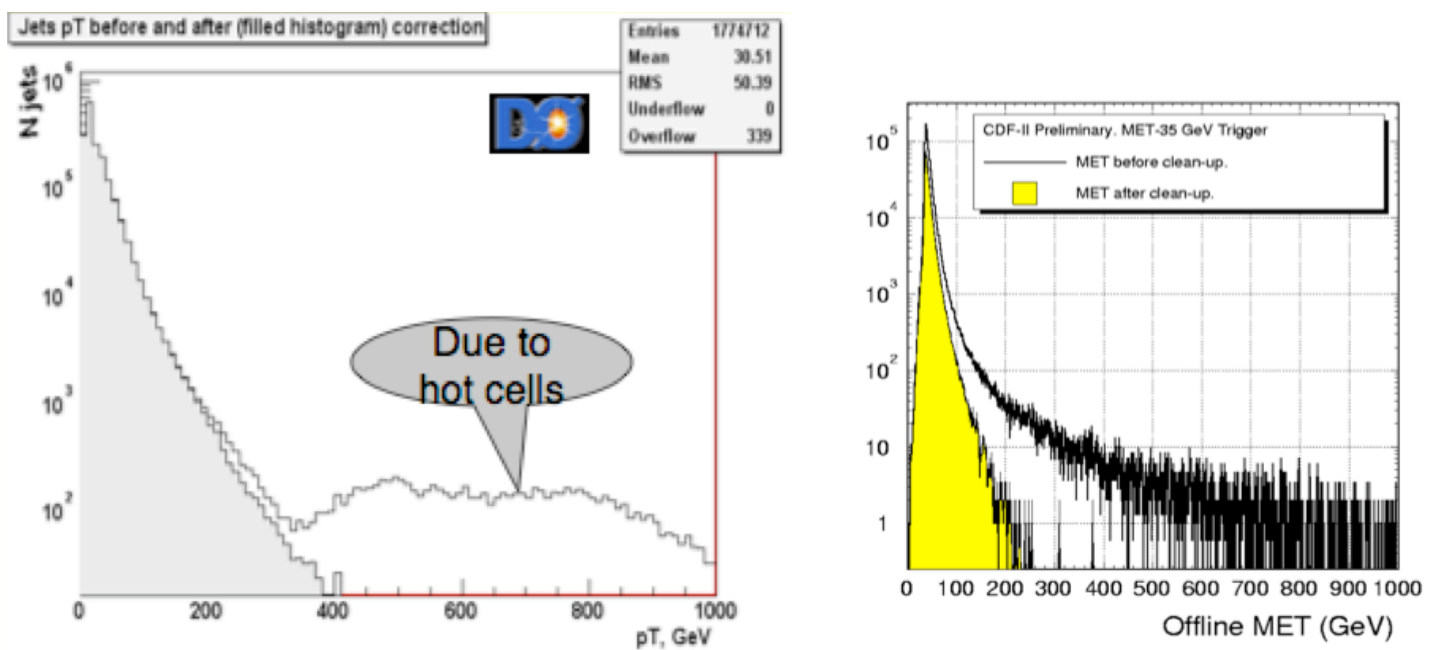

Fig. 4. Left: the jet $E_{\mathrm{T}}$ spectrum in D0, before and after removal of the so-called it hot-cells, namely additional detector noise adding energy to the jet reconstruction. Right: the missing $E_{\mathrm{T}}$ spectrum at $\mathrm{CDF}$, before and after the clean-up of all sources of spurious energy deposits. 


\subsection{Theoretical tools for SM backgrounds}

A rich arsenal of theoretical tools is available today to address these tasks. Next-to-leadingorder (NLO) calculations exist for processes of interest with up to 3 final state particles, and are available in the form of codes that allow to implement basic analysis cuts at the partonic level, such as jet $E_{\mathrm{T}}$ thresholds, or rapidity cuts: production of 2 [28-33] and 3 jets [34-36], heavy quark pairs [37-39] (possibly with an extra jet [40]), associated production of electroweak gauge bosons and one or two jets $[41,42]$, possibly including heavy quarks $[43,44]$. Next-tonext-to-leading-order (NNLO) results are available for lower multiplicities final states, most notably inclusive Drell-Yan [45] and Higgs production [46, 47]. Also in this case, calculations exist allowing for explicit cuts to be placed on the final state particles, simulating more closely the impact of experimental analyses [48-51].

Such parton-level calculations make it possible to predict inclusive quantities, and to accurately benchmark the absolute production rates. This serves the dual purpose of assessing the stability and reliability of the leading-order (LO) calculations typically used in the full event generators, and of allowing the best possible determination of the properties (cross-sections and couplings) of the new particles being discovered. Several developments [52-54] have also solved the challenge of incorporating exact NLO calculations in the shower Monte Carlos [55, 56], leading to complete NLO shower MC codes [57-59].

The backgrounds to processes such as supersymmetric particle production, on the other hand, feature the presence of many jets in the final states. NLO calculations for such final states are still beyond feasibility, and one needs to resort to event generators capable of describing final states with large jet multiplicity, and of returning events where the full set of final hadrons, including the description of the evolution of the proton fragments, in order to simulate as accurately as possible the way a given class of processes will appear inside the detector. The goal in this case is not necessarily a first-principle predictivity, but to achieve, possibly after tuning against the data, a good agreement with the data.

To achieve this, our calculations need to describe as accurately as possible both the full matrix elements for the underlying hard processes, as well as the subsequent development of the hard partons into jets of hadrons. However, for the complex final-state topologies we are interested in, no factorization theorem exists to rigorously separate these two components. The main obstacle is the existence of several hard scales, like the jet transverse energies and di-jet invariant masses, which for a generic multi-jet event will span a wide range. This makes it difficult to unambiguously separate the components of the event, which belong to the "hard process" (to be calculated using a multi-parton amplitude) from those developing during its evolution (described by the parton shower). A given $(n+1)$-jet event can be obtained in two ways: from the collinear/soft-radiation evolution of an appropriate $(n+1)$-parton final state, or from an $n$-parton configuration where hard, large-angle emission during its evolution leads to the extra jet. A factorization prescription (in this context this is often called a "matching scheme" or "merging scheme") defines, on an event-by-event basis, which of the two paths should be followed. The primary goal of a merging scheme is therefore to avoid double counting (by preventing some events to appear twice, once for each path), as well as dead regions (by ensuring that each configuration is generated by at least one of the allowed paths). Furthermore, a good merging scheme will optimize the choice of the path, using the one that guarantees the best possible approximation to a given kinematics. Different merging schemes have been proposed [60-65], all avoiding the double counting and dead regions, but leading to different results in view of the different ways the calculation is distributed between the matrix element and the shower evolution. As in any factorization scheme, the physics is independent of the separation between phases only if we have complete control over the perturbative expansion. Otherwise a residual scheme-dependence is left. Exploring different merging schemes is therefore one of the elements necessary to assess the systematic uncertainties of multi-jet calculations.

In the next sections we shall review, one by one, the issues that emerge when considering each of the background sources introduced above, and the current status of the theoretical predictions, describing, where possible, the validation tests that can be performed today either comparing different calculations, or comparing against the available Tevatron data. 


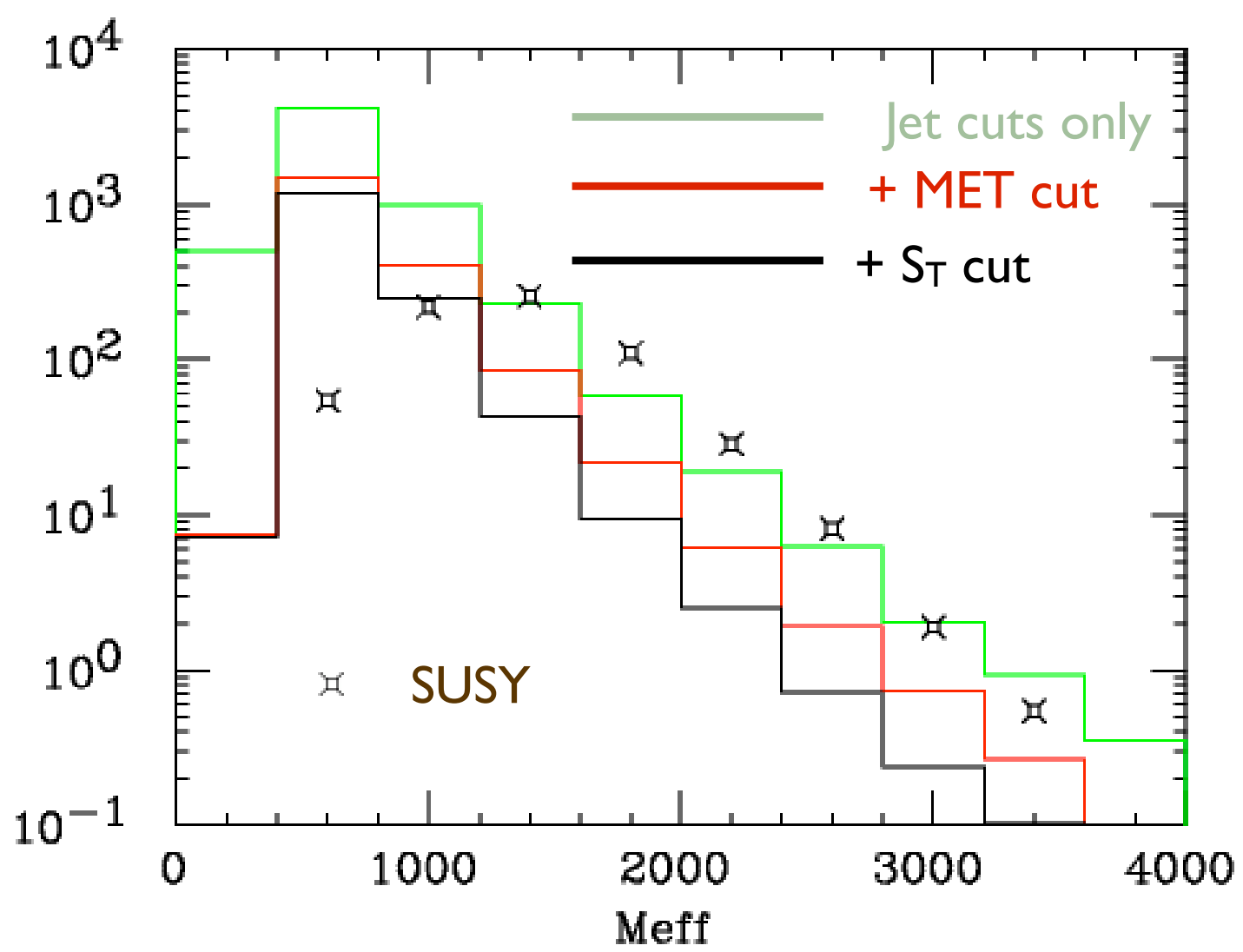

Fig. 5. Distribution of the $M_{\text {eff }}$ variable for the $Z+4$ jet process, with $Z \rightarrow \nu \bar{\nu}$. The different histograms represent the evolution of the background when additional signal cuts are imposed to the final state.

\section{$4 Z$ +jets}

We start from the only irreducible background, namely $(Z \rightarrow \nu \bar{\nu})+4$ jets. Its size, compared to the supersymmetry signal extracted from fig. 2 , is shown in fig. 5 , after the sequential application of the analysis cuts. This comparison, as well as those that will be shown in the subsequent sections, is only indicative since, while the signal is derived from a full detector simulation, the background was obtained by applying simple particle-level cuts to the output of the event generator [66]. The main purpose of this study is therefore to show the relative size of the different background components, and their evolution as a function of the cuts.

The uppermost histogram corresponds to the application of just the $E_{\mathrm{T}}$ cuts to the 4 jets. The following curves correspond to imposing the cut to the $E_{T}$ (namely to the $p_{\mathrm{T}}$ of the $Z$ boson), and to the transverse sphericity, $S_{T}$. No veto against leptons is required, since there are no leptons in this final state. Notice that, while the absolute background rate after all cuts is only about $10 \%$ of the signal, the shapes of the two in the large- $M_{\text {eff }}$ region are very similar. How reliable is this absolute normalization? Figure 6 shows the important difference in rate obtained using a shower MC to generate the jets from the shower evolution, and using a full matrix-element calculation. As the jet multiplicity grows, the shower approach underestimates the cross section more and more. While the matrix-element approach is certainly more reliable, this comparison underscores the possibly large systematics of a theoretical calculation for such complex final states. The validation against the data is therefore a necessary step before any application of the theoretical predictions. Confidence in the reliability of the matrix element calculations comes from the measurements of the Tevatron experiments of $Z+$ multijet final states, with $Z \rightarrow \ell^{+} \ell^{-}$. CDF [67] has compared the $Z+1$ and 2 jet rates against the NLO 


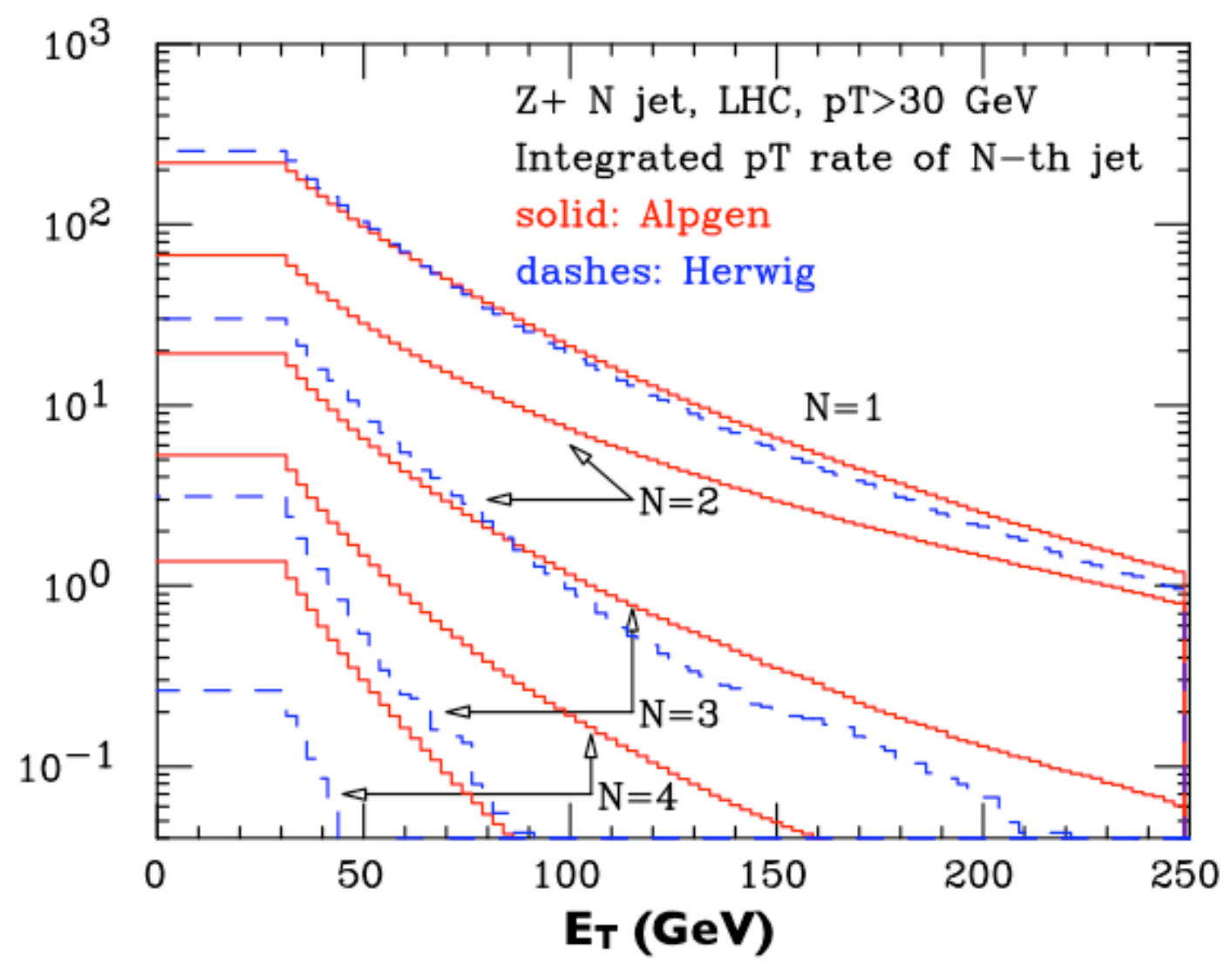

Fig. 6. Integrated $E_{\mathrm{T}}$ spectrum of the $N$-th jet $(N=1,2,3,4)$ in $Z+N$-jet events, as derived from the shower monte carlo HERWIG (dashes) and from a leading-order matrix-element calculation with the monte carlo ALPGEN (solid).

calculations of [41], finding excellent agreement in both normalization and in shapes, as shown in fig. 7. The cross section for multijet production has been measured by D0 [68], and compared against the NLO results for 1 and 2 jets, and against LO matrix elements [64] for up to 4 jets, as shown in fig. 8

Once the LHC data will be available, the measurement of the $\left(Z \rightarrow \ell^{+} \ell^{-}\right)+$jets rates can be used to validate the extrapolation of the theoretical descriptions from the energy of the Tevatron to that of the LHC. Few hundred $\mathrm{pb}^{-1}$ of integrated luminosity will be enough to determine the overall normalization in the region of $M_{\text {eff }}$ dominated by the background (below $1 \mathrm{TeV}$ ). Assuming that the shape of the theoretical prediction is reliable, this will be enough to extend the background estimate at the higher values of $M_{\text {eff }}$.

\section{$5 W+$ jets}

The processes $W+$ jets and $Z+$ jets are very similar from the point of view of QCD. There are minor differences related to the possibly different initial-state flavour compositions, but the main theoretical systematics, coming from the renormalization-scale sensitivity due to the lack of higher-order perturbative corrections, are strongly correlated. It is therefore reasonable to assume that the clean measurement of $Z+$ jets, with $Z \rightarrow \ell^{+} \ell^{-}$, should be sufficient to validate also the calculations of the $W+$ jet rates. It is nevertheless useful to understand the size and features of this contribution. This is shown in fig. 9 for the $[W \rightarrow \ell \nu]+4$-jet final states, and in fig. 10 for the $[W \rightarrow \tau \rightarrow$ hadrons $]+3$-jet final states. 

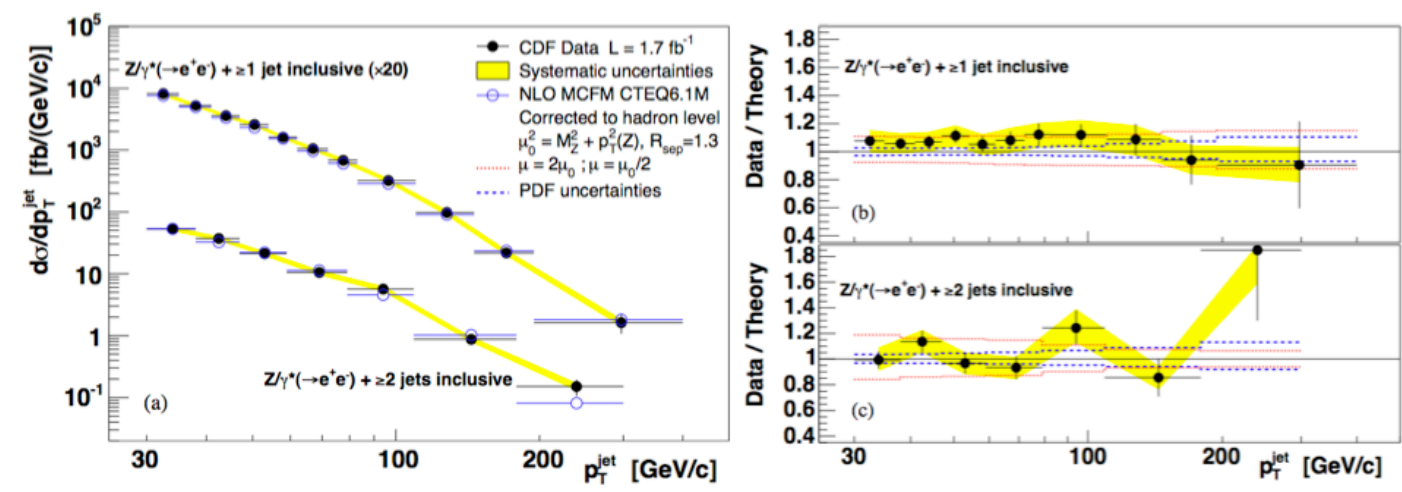

Fig. 7. Leading-jet $E_{\mathrm{T}}$ spectrum in $Z+\geq 1$ - and $Z+\geq 2$-jet events, as measured by CDF at the Tevatron [67], compared against the next-to-leading-order theoretical calculation from the monte carlo MCFM [41].

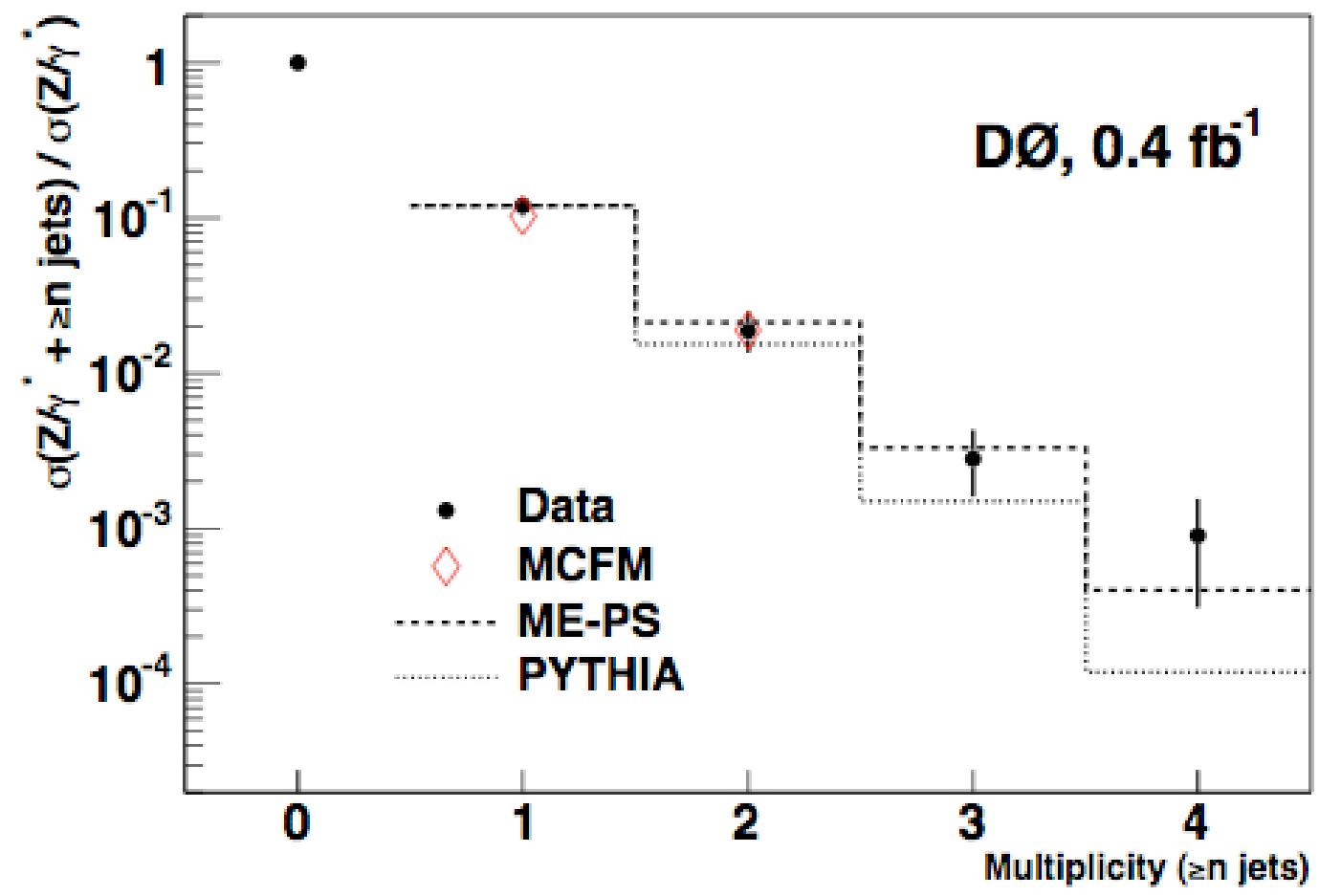

Fig. 8. Cross section for the production of $Z+$ multijet final states, as measured by D0 [68], compared against the next-to-leading-order [41] and the leading-order matrix-element plus shower calculations [64]. 


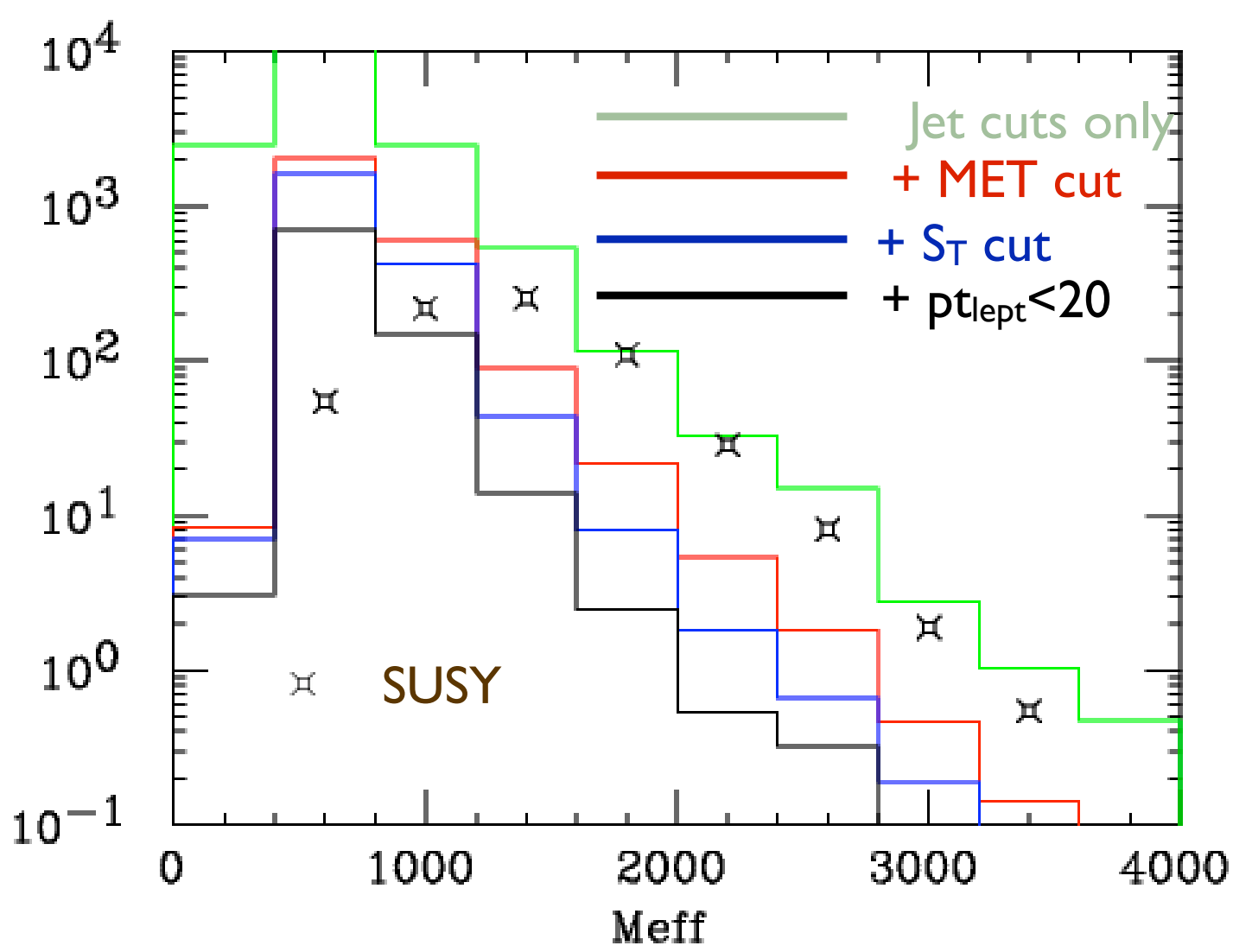

Fig. 9. Distribution of the effective mass variable, $M_{\text {eff }}$, for the $W+4$ jet process, with $W \rightarrow \ell \nu$ $(\ell=e, \mu)$. The different histograms represent the evolution of the background when additional signal cuts are imposed to the final state.

The validation of the theoretical predictions for the Tevatron has been established by a recent measurement at CDF [69]. The ratio of the measured and predicted $W+N$-jet cross sections, for jets with $E_{\mathrm{T}}>25 \mathrm{GeV}$, is shown in Fig. 11. The theoretical predictions include the LO results from Ref. [66] (labeled as MLM), and from Ref. [64] (labeled as SMPR), while MCFM refers to the NLO predictions for the 1- and 2-jet rates from ref. [41]. The systematic uncertainties of the individual calculations, mostly due to the choice of renormalization scale, are shown. The LO results, which have an absolute normalization for all $N$-jet values, are in good agreement with the data, up to an overall $K$ factor, of order 1.4. The prediction for the ratios of the $N$-jet and $(N-1)$-jet rates is also in good agreement with the data. The NLO calculations embody the $K$ factor, and exactly reproduce the 1- and 2-jet rates.

Once again, the LHC data will allow a direct check of the correctness of the theoretical estimates. It should be stressed, however, that the measurement of the $W+$ jets cross section, required to validate the theoretical calculations, will not be easy. The Tevatron experience has shown, in fact, that important backgrounds contaminate the $W+$ jet samples. For example, large contributions [69] come from the so-called non- $W$ backgrounds, where the lepton and missing transverse energy do not originate from a $W$ decay. Possible sources of such processes include heavy quark (charm and bottom) production, where the lepton originates from an isolated semileptonic decay of the heavy quark, and the missing energy from jet mismeasurements and from the decay to a hard neutrino of the heavy antiquark. Furthermore, for large jet multiplicity, a large fraction of the $W+$ jet signal comes from $t \bar{t}$ decays, an effect that will be even more pronounced at the LHC (perversely enough, it can be argued [70] that supersymmetry itself could contaminate the measurement of the SM background processes!). Therefore, the 


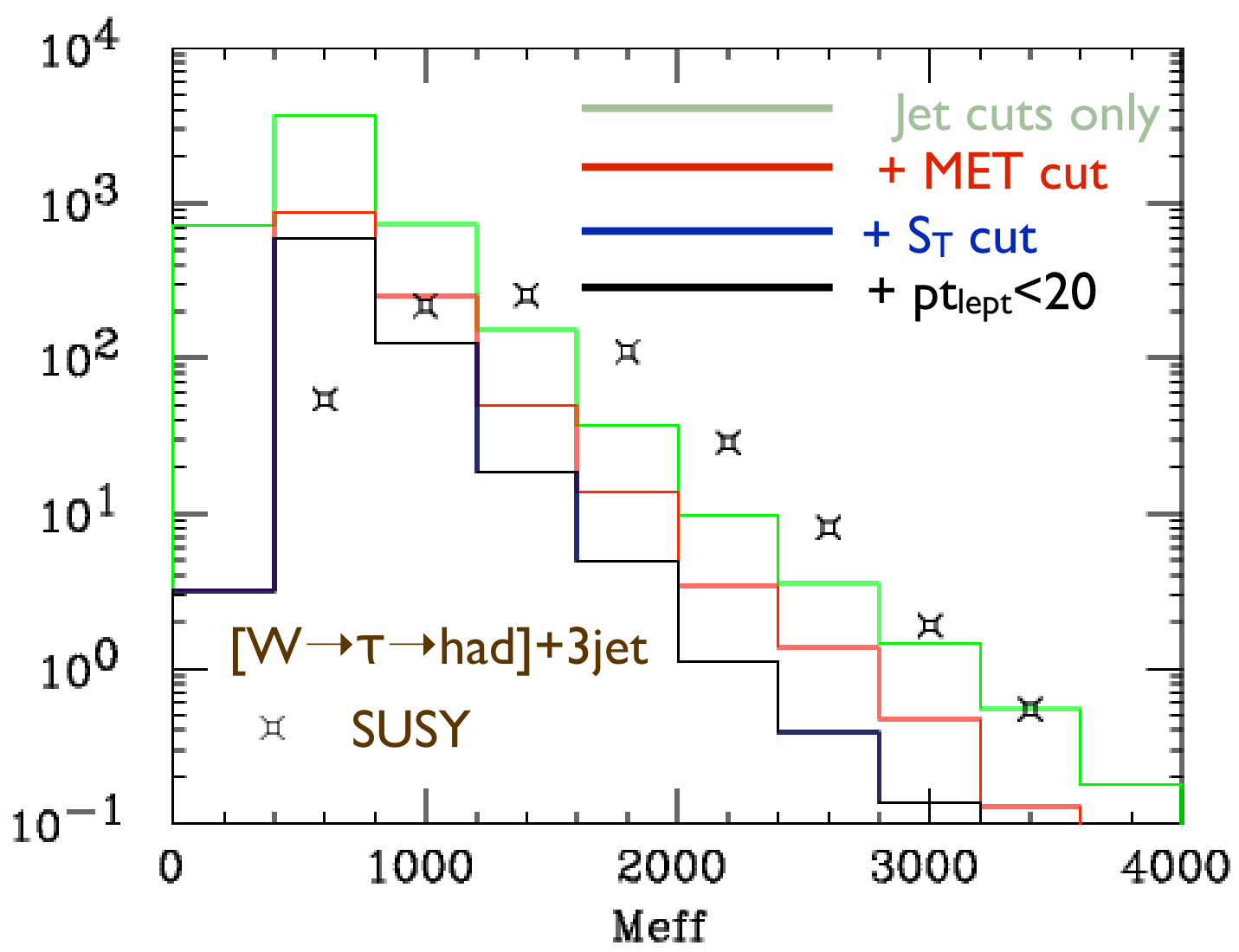

Fig. 10. Distribution of the effective mass variable, $M_{\mathrm{eff}}$, for the $W+3$ jet process, with $W \rightarrow \tau \rightarrow$ hadrons. The different histograms represent the evolution of the background when additional signal cuts are imposed to the final state.

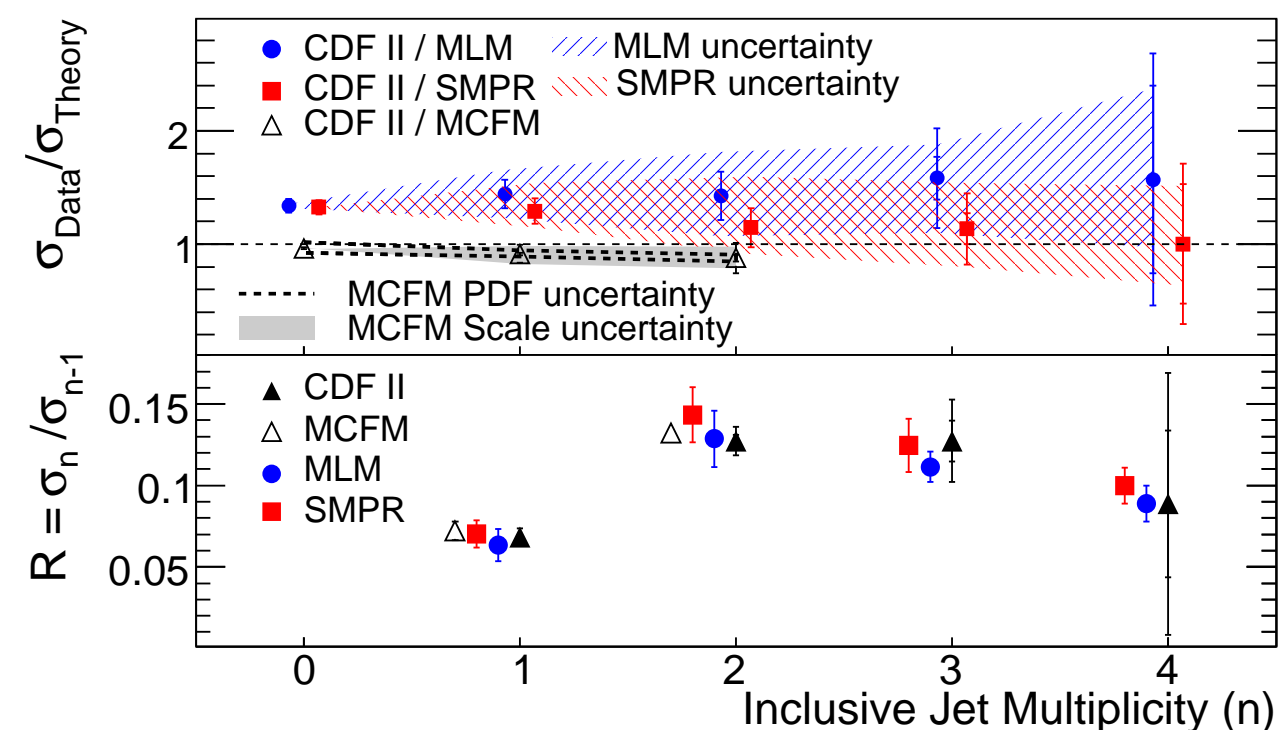

Fig. 11. Comparison between CDF data and theory for $W+N$ jets cross sections [69] . 

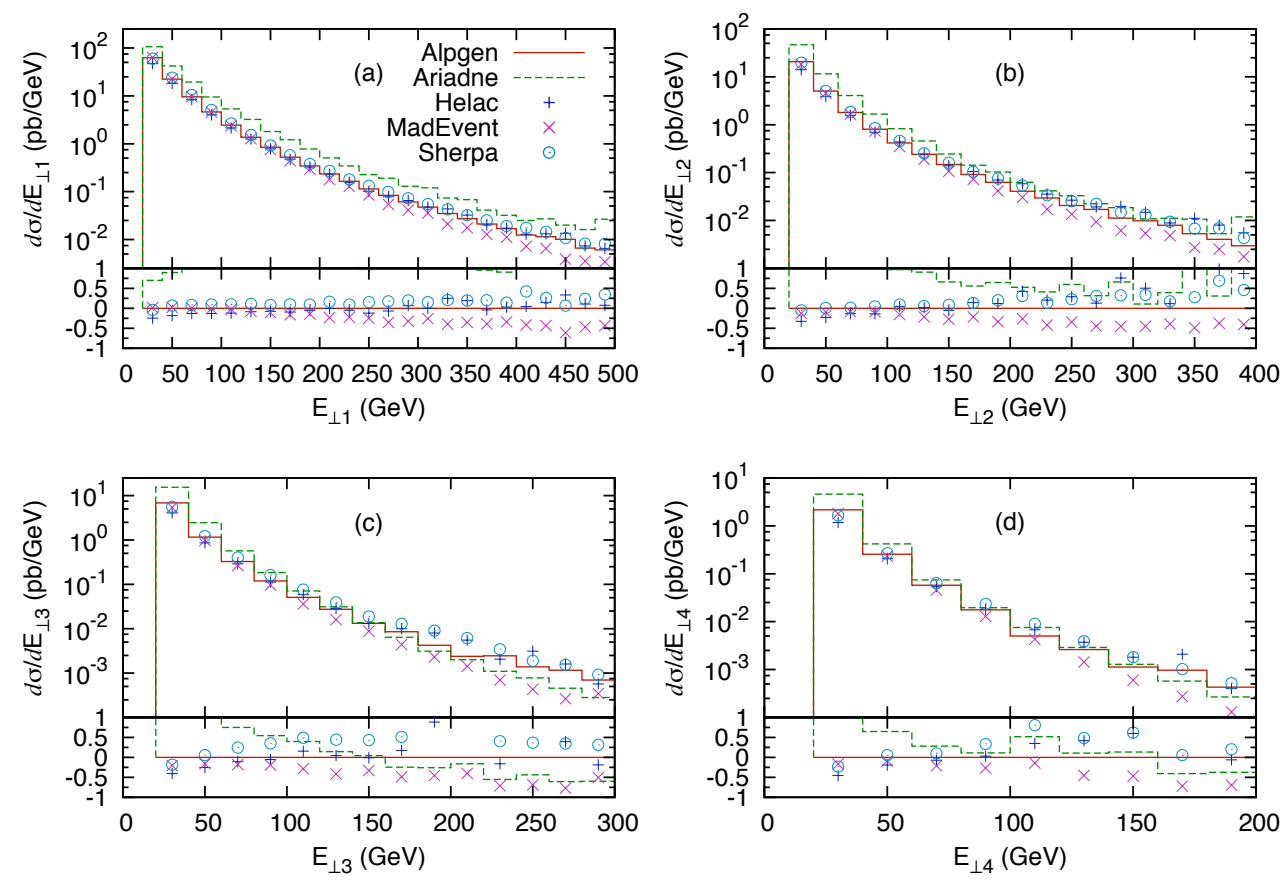

Fig. 12. Predicted jet $E_{\mathrm{T}}$ spectra in $W+$ jet(s) final states at the LHC [65]

background to supersymmetry signals has itself its own backgrounds, and its accurate determination will provide a challenge by itself. It is difficult to anticipate the scale of the challenge, only the direct contact with the data will tell!

\subsection{Theoretical status of $W+$ multijet final states}

Due to the importance of $W+$ multijet processes as a background to many analyses, a significant effort has been invested recently in its theoretical understanding. As mentioned above, NLO calculations are available for the 1- and 2-jet final states [41]. These calculations are not available in the form of full MC event generators, describing the complete structure of the final states, but provide a reference benchmark for the results of calculations based on LO matrix elements, merged with the full shower evolution. Several groups [66,71-75] have developed tools to extend the LO predictions to high jet multiplicity, addressing the problem of merging the higher order processes with the shower evolution without double counting. The double counting problem refers to the multiple covering of same phase-space configurations when a jet can be generated both by the direct matrix element calculation of a $N$-jet configuration and by the possible hard radiation of a jet during the shower evolution of a $(N-1)$-parton final state. Thorough comparisons have been performed [65] between the predictions of these tools, resulting in a reliable framework for the estimates of systematic uncertainties. The results of the matrix element evaluation for these complex processes are all in excellent agreement; differences in the predictions at the level of hadrons may arise from the use of different parton-shower approaches, and of different ways of sharing between matrix elements and shower the task of describing the radiation of hard jets (merging algorithms). An example of the spread in the different predictions is shown in Fig. 12, which shows the $E_{\mathrm{T}}$ spectra of the four highest- $E_{\mathrm{T}}$ jets in $W+$ multijet events at the LHC. With the exception of the predictions from one of the codes, all results are within $\pm 50 \%$ of each other, an accuracy sufficient by itself to establish possible deviations such as those in Fig. 2. 

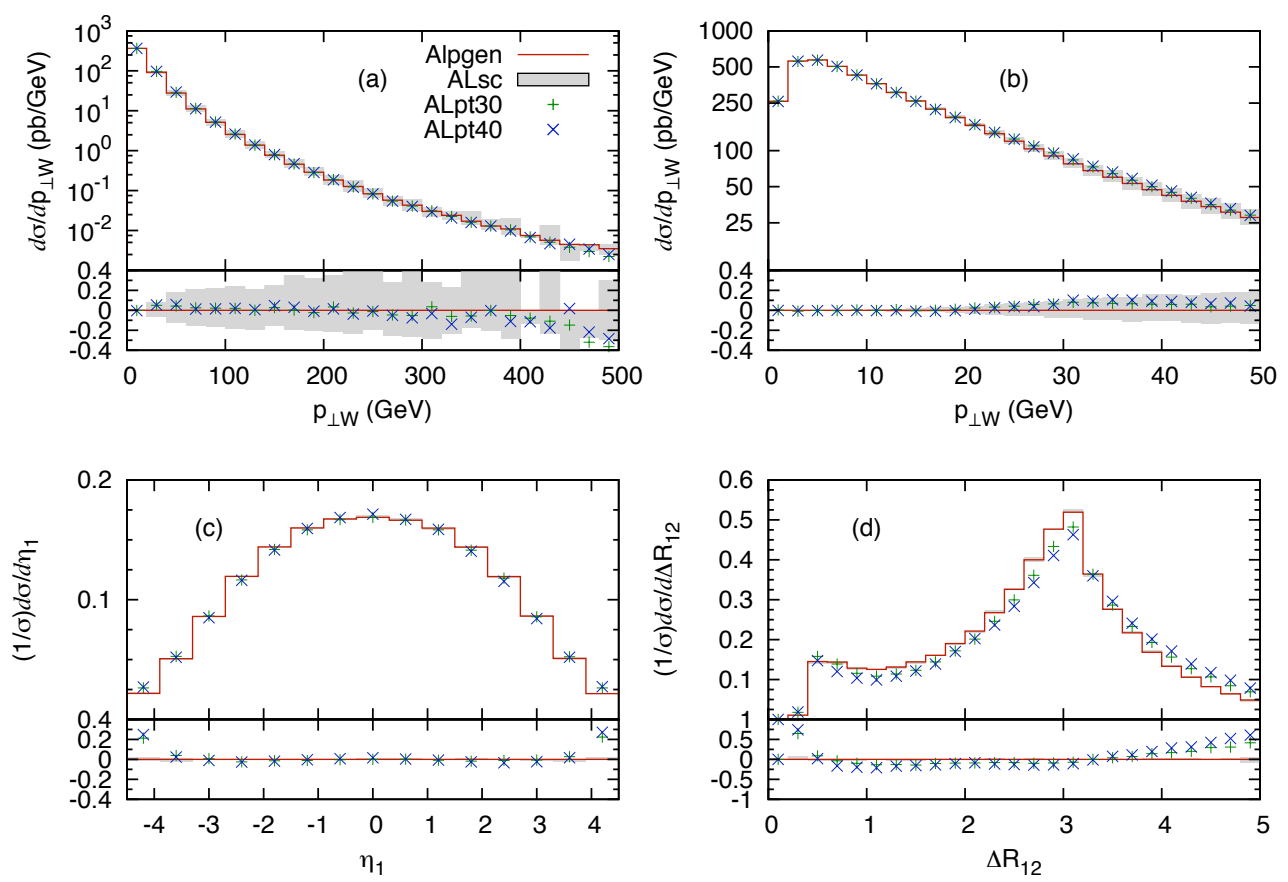

Fig. 13. Systematic uncertainty for a few $W+$ multijet observables, estimated from the ALPGEN calculations [65].

These differences are of size compatible with the intrinsic uncertainties of the calculations, given for example by the size of the bands in Fig. 11. An alternative picture of the intrinsic systematics of the ALPGEN predictions for observables like the $p_{\mathrm{T}}$ spectrum of the $W$ boson, the leading-jet pseudorapidity distribution, and the angular correlations between the two leading jets, is given in fig. 13 . Similar results are obtained for the other codes [65]. It is expected that these systematics can be reduced by tuning the input parameters, like the choice of renormalization scale, by fitting the data. An accurate determination of the normalization and shape of the SM background to a supersymmetric signal could therefore be obtained by analyzing data control samples. A clear path is therefore available to establish the accuracy of the theoretical tools, and to provide robust background estimates for searches of anomalies in the multijet plus $E_{T}$ final states.

\section{$6 t \bar{t}$ production}

With a total cross section of order $1 \mathrm{nb}$, and a rich set of final states including leptons, missing energy, jets and heavy quarks [76-78], the production of top quarks at the LHC [79] is one of the potentially largest backgrounds to almost any type of new phenomena. In the case of the multijet $+\not_{T}$ signature of supersymmetry, one requests one of the two $W$ bosons from the $t \bar{t}$ to decay leptonically, and the other hadronically, leading to a characteristic 4-jet plus $\mathbb{E}_{T}$ final state. Early studies of $t \bar{t}$ final states, nevertheless, suggested that this contribution would be very small. Considering only the lowest-order process $p p \rightarrow t \bar{t}$, it is easy to understand why it should be so. The request of a large value of $M_{\text {eff }}$, in fact, forces the production kinematics into the region where the $t$ and $\bar{t}$ recoil against each other at very large $p_{\mathrm{T}}$. This kinematics leads (see upper panel in fig. 14) to several consequences: on one side the request for a large $\not_{T}$ forces the $W$ to be highly boosted, thus typically leading to a high- $p_{\mathrm{T}}$ lepton as well. On the other, the "3-jets" coming from the hadronic top decay would coalesce into a single fat one, and the request of having 4 separate jets would not be met. This implies that, in order to satisfy the 


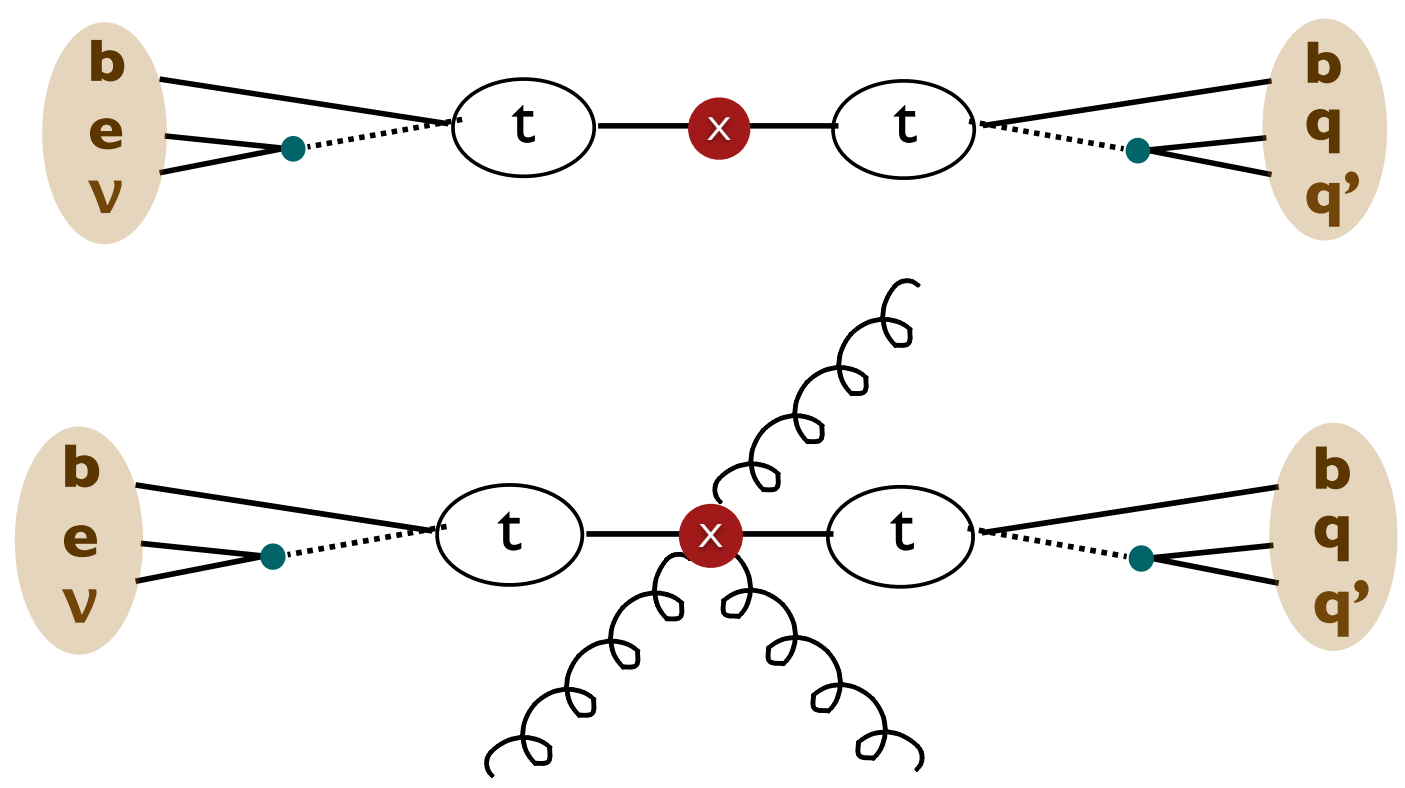

Fig. 14. $t \bar{t}$ final state at large $p_{T}$.

supersymmetry-selection cuts, one needs additional hard jets not coming from the top decays (see lower panel of fig. 14). The dominance of these higher-order processes is confirmed by the calculation, as shown in fig. 15, where we present the $M_{\text {eff }}$ spectra of events with 1,2 and 3 hard partons in addition to the $t \bar{t}$ decays products. Notice that these high-jet-multiplicity final states would typically be underestimated by the standard parton-shower approximation. As in the case of the $Z / W+$ multijet final states, a reliable background estimate requires therefore the use of higher-order matrix element codes.

\subsection{Theoretical status of $t \bar{t}+$ multijet final states}

The theoretical prediction for inclusive $t \bar{t}$ production have already been well tested at the Tevatron [80-82]. For example, NLO calculations [37,38], enhanced by the resummation of leading [83] and subleading Sudakov logarithms [84-86] or by the inclusion of classes of NNLO terms [87-89] predict the total cross section with an accuracy consistent with the experimental uncertainty [90-98], as shown by fig. 16. The predictions for the LHC are expected to be equally accurate $[86,88,89]$, if not more, since the main source of uncertainty, the parton distribution functions, fall at the LHC in a range of $x$ values where they are known with precision better than at the Tevatron $[99,100]$. Recent progress towards a complete NNLO calculation [40,101-105] will likely push the ultimate accuracy of the total cross section to the level of few percent.

The extreme kinematics of $t \bar{t}$ final states responsible for the background to supersymmetry searches, however, cannot be tested at the Tevatron, due to the limited statistics and phasespace. It is only with the LHC data that compelling tests will become possible. Except for $t \bar{t}+1$ jet final states [40], predictions for the associated production of $t \bar{t}$ and several jets are only available using LO matrix element calculations, merged with parton shower evolution codes. Where possible, these LO tools have been validated against the existing NLO+shower descriptions. The NLO corrections to $t \bar{t}$ production have been incorporated $[58,59]$ in fact in the HERWIG [55] and PYTHIA [56] Monte Carlos, allowing for a complete description, at NLO accuracy, of the inclusive $t \bar{t}$ final states. Predicted properties such as the transverse momentum distribution or the invariant mass of the $t \bar{t}$ pair, agree very well with those extracted from the LO calculations merged with parton showers [63], as shown in fig. 17. Differences between the 


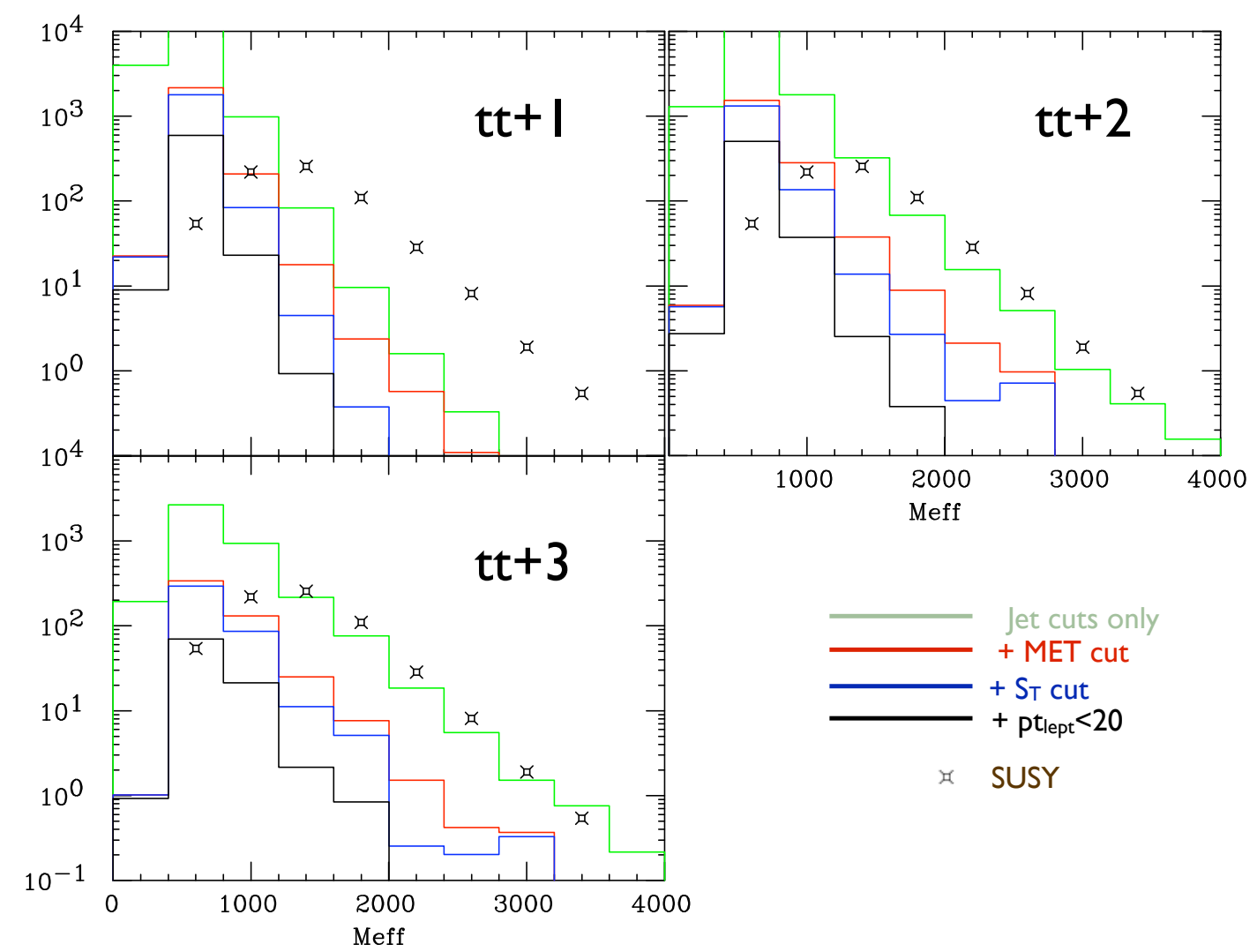

Fig. 15. Contributions to the $M_{\text {eff }}$ distribution coming from production of $t \bar{t}$ pairs in association with 1, 2 and 3 extra hard partons.
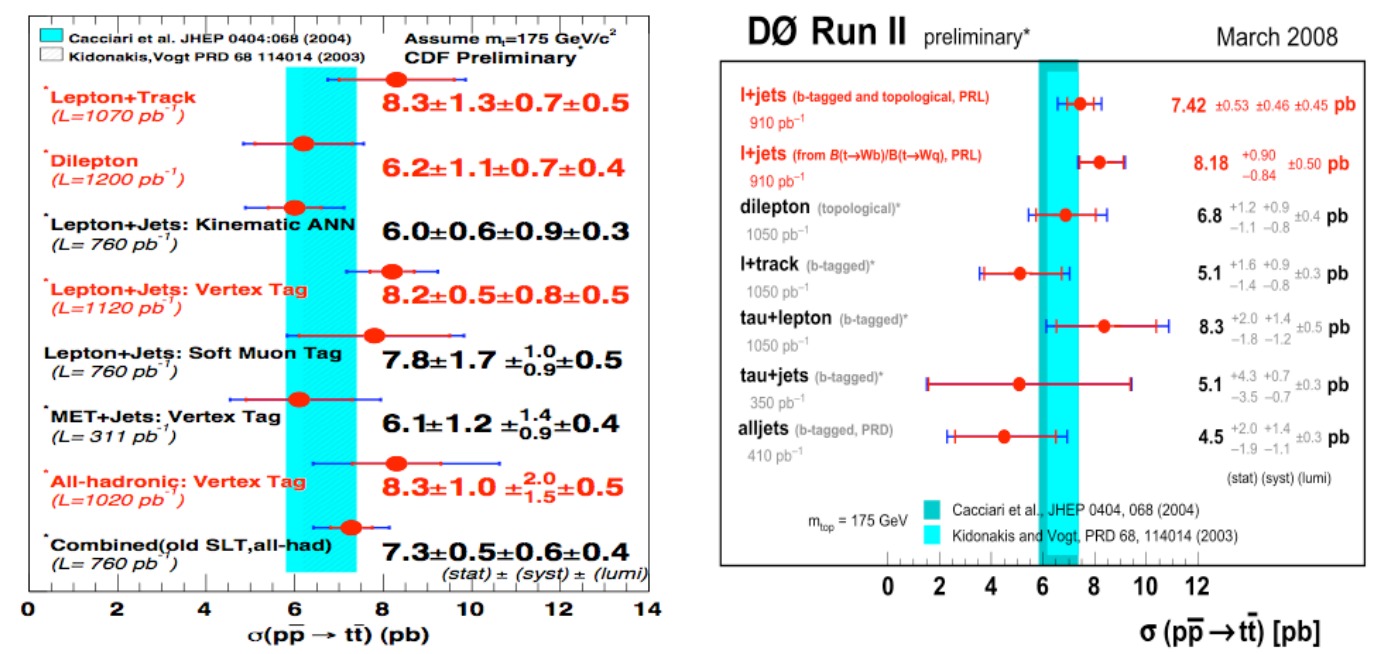

Fig. 16. $t \bar{t}$ cross section measurements at the Tevatron from CDF (left) and D0 (right). Summary taken from [81]. 

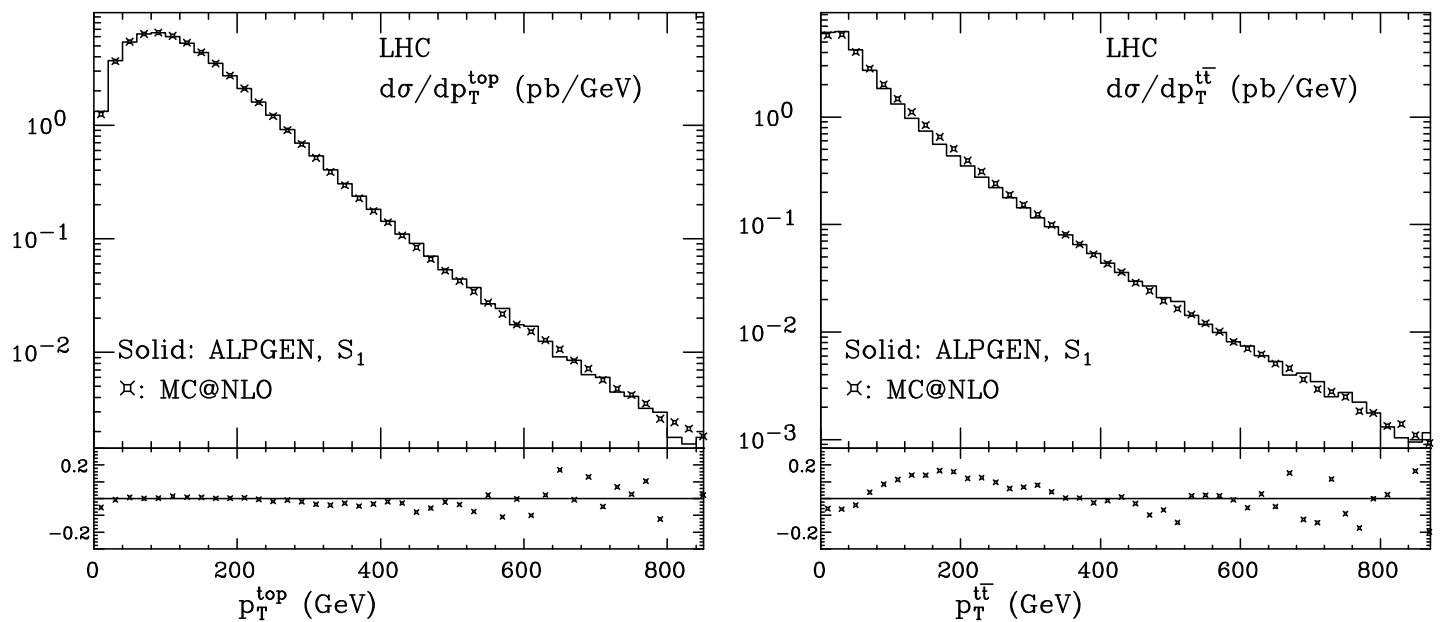

Fig. 17. Inclusive spectrum of the top quark (left), and $p_{\mathrm{T}}$ of the $t \bar{t}$ pair (right) as described by the MC@NLO (points) and by ALPGEN LO+HERWIG calculations.

two calculations emerge when considering the production rate of several additional jets, but remain relatively modest, as shown in fig. 18. Specific studies dealing with the high- $p_{\mathrm{T}}$ regions characteristic of the supersymmetry searches, however, have not been carried out as yet. Once again, only the direct comparisons with the LHC data will provide the definitive validation of these calculations.

\section{QCD multijets}

The large energy available in LHC collisions can allow for the loss of significant amounts of transverse energy via jets emitted at very large rapidity, as shown in fig. 19. A quantitative estimate of this effect is shown in fig. 20. We show here the ratio

$$
\frac{\sigma_{j j}\left(E_{T}>E_{T 0} ;|\eta|>\eta_{\max }\right)}{\sigma_{p p}}
$$

where $\sigma_{p p}$ is the total $p p$ cross section, and the numerator is the cross section to produce a dijet pair, with one of the two jets escaping detection due to its large pseudorapidity $|\eta|>\eta_{\max }$. These final states would appear as a 1-jet event, with missing $E_{\mathrm{T}}$. If such events were to overlap with a 3 -jet event, they would lead to a 4 -jet $+\mathbb{E}_{T}$ signature, faking the supersymmetry signal. At high luminosity, the large number of additional $p p$ interactions would amplify the probability that one such jet $+\mathbb{E}_{T}$ event overlapped with whatever primary multijet final state. The results of fig. 19 show that a calorimetric coverage out to $\eta$ of order 3 of 4 would lead to a large $\not_{T}$ signal. At the highest expected luminosities, $L \sim 10^{34} \mathrm{~cm}^{-2} \mathrm{~s}^{-1}$, the number of overlapping events is of the order of 20 , and the probability of $E_{T} \gtrsim 100 \mathrm{GeV}$ can become of $\mathrm{O}\left(10^{-4}\right)$, thus leading to a background as large as the signal. For this reason the LHC calorimeters extend out to about $\eta=5$. The current theoretical calculations of multijet final states are based on LO matrix elements, merged with shower MC. In the case of ALPGEN, this extends out to multiplicities of about 6 , thus suitable for the study of supersymmetry backgrounds. These calculations were tested during the run 1 of the Tevatron, showing a good agreement [106]. The early LHC data taking, with a low luminosity and a small number of overlapping $p p$ collisions, will provide a robust validation of the calculations. Other instrumental sources of $\mathbb{E}_{T}$, as e.g. the non-gaussian tails of the jet-energy resolution, will be monitored studying the energy balance of $\gamma$-jet and $Z$-jet events, as discussed in the case of the Tevatron analyses in ref. [107]. 


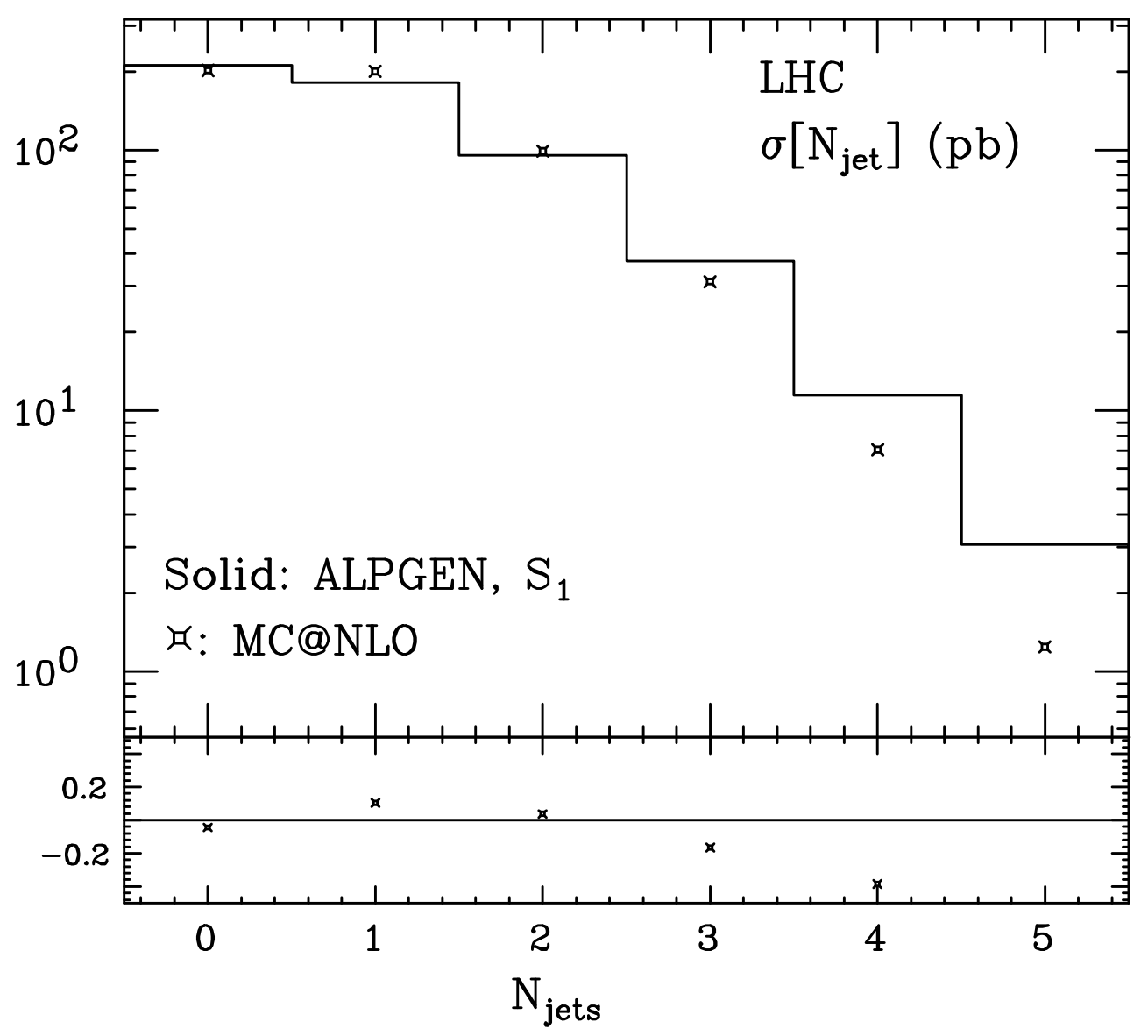

Fig. 18. Multiplicity of jets associated to $t \bar{t}$ pairs, as described by the MC@NLO (points) and by ALPGEN leading-order+HERWIG calculations.

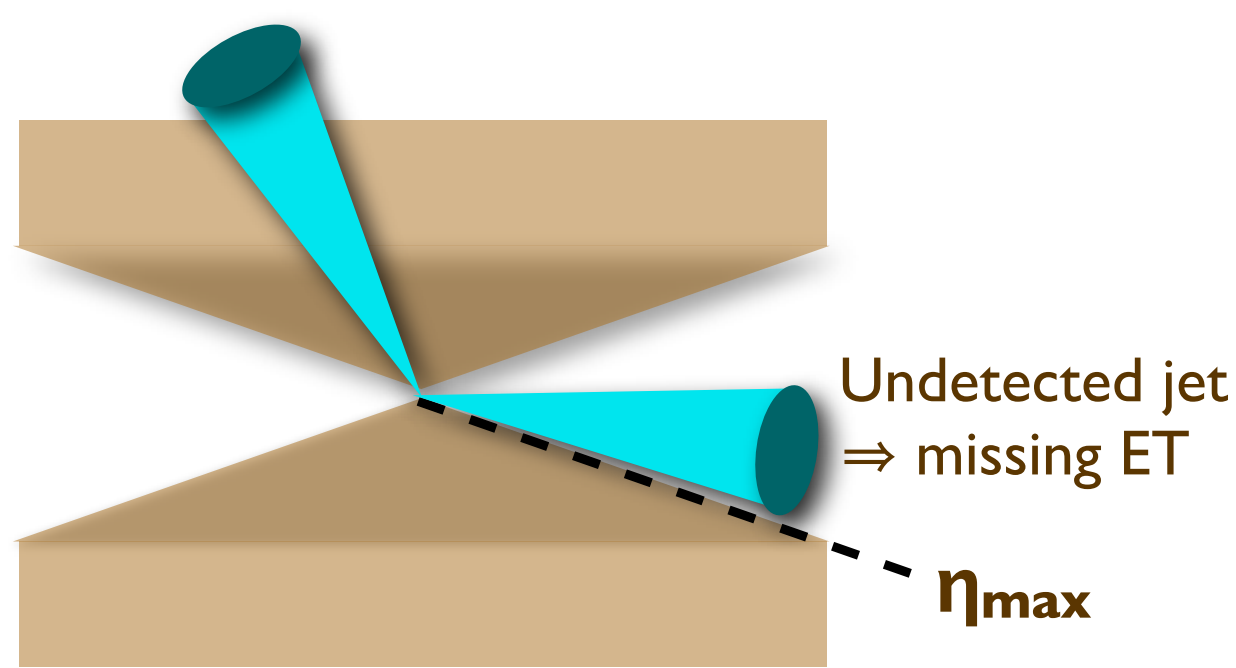

Fig. 19. Limited calorimetric coverage as a source of missing transverse momentum in multijet final states. 


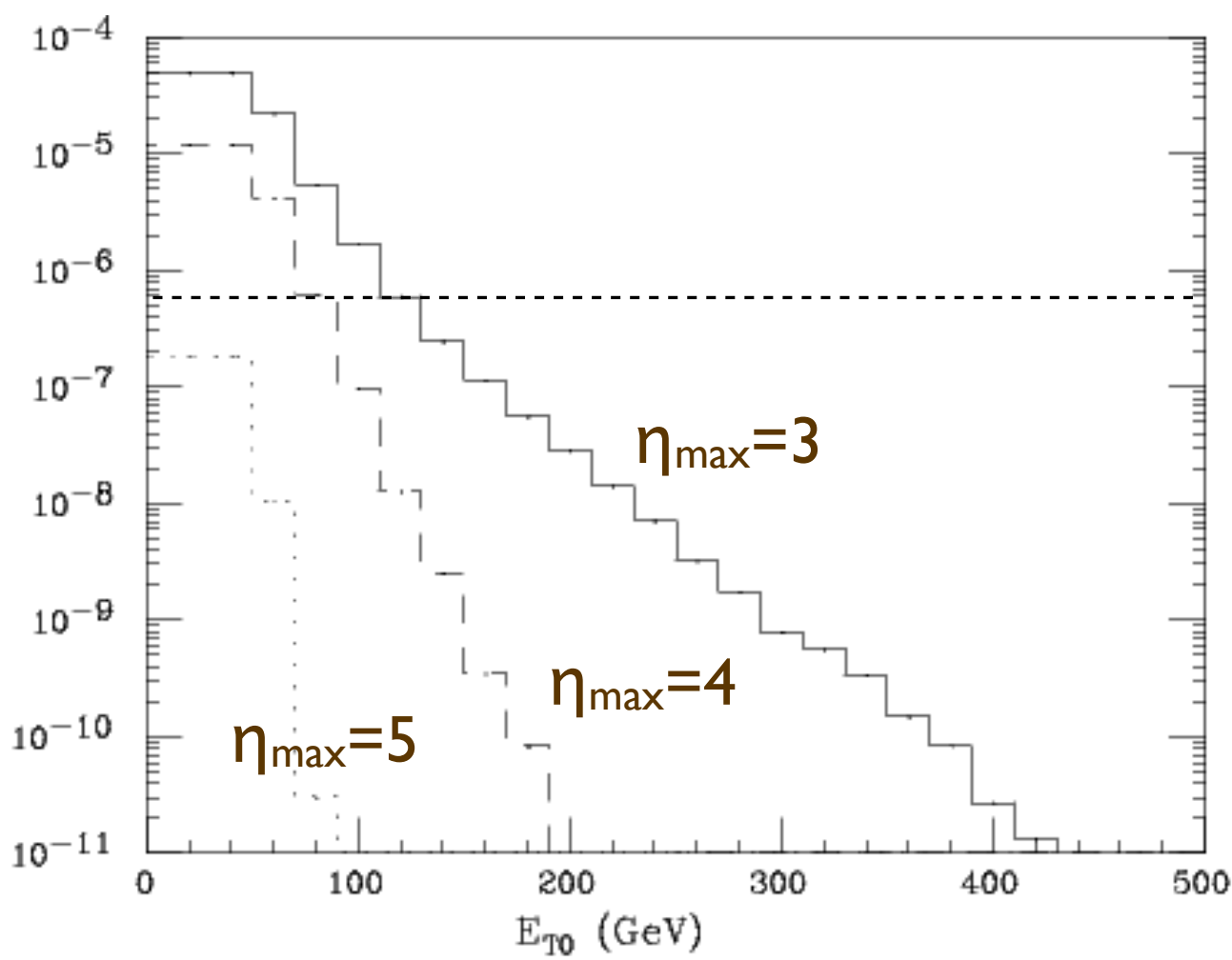

Fig. 20. Fraction of the total $p p$ cross section with missing transverse energy $\not_{T}>E_{T 0}$, due to the loss of a jet at pseudorapidity $\eta>\eta_{\max }$, for various values of $\eta_{\max }$. The horizontal dashed line corresponds to the rate of inclusive $W$ events decaying leptonically.

\section{Conclusions}

Advanced MC tools for the description of the SM, and for the isolation of possible new physics at the LHC, are becoming mature. Validation and tuning efforts are underway at the Tevatron, and show that a solid level of understanding of even the most complex manifestations of the SM are well under control. The extrapolation of these tools to the energy regime of the LHC is expected to be reliable, at least in the domain of expected discoveries, where the energies of individual objects (leptons, jets, missing energy) are of order $100 \mathrm{GeV}$ and more. However, the consequences of interpreting possible discrepancies as new physics are too important for us to blindly rely on our faith in the goodness of the available tools. An extensive and coherent campaign of MC testing, validation and tuning at the LHC will therefore be required. Its precise definition will probably happen only once the data are available, and the first comparisons will give us an idea of how far off we are and which areas require closer scrutiny.

Ultimately the burden, and the merit, of a discovery should and will only rest on the experiments themselves! The data will provide the theorists guidance for the improvement of the tools, and the analysis strategies will define the sets of control samples that can be used to prepare the appropriate and reliable use of the theoretical predictions.

Aside from the discovery of anticipated objects like the $W, Z$ and the top, we have never faced with high-energy colliders the concrete situation of a discovery of something beyond the expected. In this respect, we are approaching what the LHC has in store for us without a true experience of discovering the yet unknown, and we should therefore proceed with great caution. All apparent instances of deviations from the SM emerged so far in hadronic or leptonic high-energy collisions have eventually been sorted out, thanks to intense tests, checks, and 
reevaluations of the experimental and theoretical systematics. This shows that the control mechanisms set in place by the commonly established practice are very robust.

Occasionally, this conservative approach has delayed in some areas of particle physics the acceptance of true discoveries, as in the case of Davies's neutrino mixing, and as might turn out to be the case for the muon anomaly. But it has never stopped the progress of the field, on the contrary, it has encouraged new experimental approaches, and has pushed theoretical physics to further improve its tools.

The interplay between excellent experimental tools, endowed with the necessary redundancy required to cross-check odd findings between different experiments and different observables, and a hard-working theoretical community, closely interacting with the experiments to improve the modeling of complex phenomena, have provided one of the best examples in science of responsible and professional modus operandi. In spite of all the difficult challenges that the LHC will pose, there is no doubt in my mind that this articulated framework of enquiry into the yet unknown mysteries of nature will continue providing compelling and robust results.

\section{Acknowledgements}

This work is supported in part by the European Community's Marie-Curie Research Training Network HEPTOOLS under contract MRTN-CT-2006-035505.

\section{References}

1. J. M. Campbell, J. W. Huston and W. J. Stirling, "Hard interactions of quarks and gluons: A primer for LHC physics," Rept. Prog. Phys. 70, 89 (2007) arXiv:hep-ph/0611148.

2. M. L. Mangano, "The saga of bottom production in proton antiproton collisions," AIP Conf. Proc. 753, 247 (2005), arXiv:hep-ph/0411020; M. Cacciari, arXiv:hep-ph/0407187; M. Cacciari et al., JHEP 0407 (2004) 033; F. Happacher, P. Giromini and F. Ptohos, Phys. Rev. D 73 (2006) 014026; D. E. Acosta et al. [CDF Collaboration], Phys. Rev. D 71 (2005) 032001.

3. F. Abe et al. [CDF Collaboration], Phys. Rev. Lett. 69 (1992) 3704; S. Abachi et al. [D0 Collaboration], Phys. Lett. B 370 (1996) 239; N. Brambilla et al. [Quarkonium Working Group], arXiv:hep-ph/0412158

4. L. J. Dixon, "Hard QCD Processes at Colliders," arXiv:0712.3064 [hep-ph].

5. M. A. Dobbs et al., "Les Houches guidebook to Monte Carlo generators for hadron collider physics," arXiv:hep-ph/0403045

6. M. L. Mangano, "Understanding the Standard Model, as a bridge to the discovery of new phenomena at the LHC," arXiv:0802.0026 [hep-ph].

7. R. M. Barnett, J. F. Gunion and H. E. Haber, "Gluino decay patterns and signatures," Phys. Rev. D 37 (1988) 1892.

8. H. Baer, C. h. Chen, F. Paige and X. Tata, "Signals for minimal supergravity at the CERN large hadron collider: Multi - jet plus missing energy channel," Phys. Rev. D 52 (1995) 2746 arXiv:hep$\mathrm{ph} / 9503271$.

9. H. Baer, C. h. Chen, F. Paige and X. Tata, "Signals for Minimal Supergravity at the CERN Large Hadron Collider II: Multilepton Channels," Phys. Rev. D 53 (1996) 6241 arXiv:hep-ph/9512383.

10. H. Baer, H. Prosper and H. Summy, "Early SUSY discovery at LHC without $\mathbb{E}_{T}$ : the role of multi-leptons," Phys. Rev. D 77 (2008) 055017 arXiv:0801.3799 [hep-ph]].

11. R. Barbieri, F. Caravaglios, M. Frigeni and M. L. Mangano, "Production And Leptonic Decays Of Charginos And Neutralinos In Hadronic Collisions," Nucl. Phys. B 367 (1991) 28.

12. Z. Sullivan and E. L. Berger, "Trilepton Production at the LHC - Standard Model Sources and Beyond," arXiv:0805.3720 [hep-ph].

13. V. M. Abazov et al. [D0 Collaboration], "Search for squarks and gluinos in events with jets and missing transverse energy using $2.1 \mathrm{fb}^{-1}$ of $p \bar{p}$ collision data at $\sqrt{s}=1.96 \mathrm{TeV}$," Phys. Lett. B 660 (2008) 449 arXiv:0712.3805 [hep-ex]].

14. A. A. Affolder et al. [CDF Collaboration], "Search for gluinos and scalar quarks in $p \bar{p}$ collisions at $\sqrt{s}=1.8 \mathrm{TeV}$ using the missing energy plus multijets signature," Phys. Rev. Lett. 88 (2002) 041801 arXiv:hep-ex/0106001. 
15. I. Hinchliffe, F. E. Paige, M. D. Shapiro, J. Soderqvist and W. Yao, "Precision SUSY measurements at LHC," Phys. Rev. D 55 (1997) 5520.

16. ATLAS detector and physics performance [ATLAS Collaboration], ATLAS-TDR-015, CERNLHCC-99-015.

17. S. Abdullin et al. [CMS Collaboration], "Discovery potential for supersymmetry in CMS," J. Phys. G 28 (2002) 469 arXiv:hep-ph/9806366.

18. G. L. Bayatian et al. [CMS Collaboration], "CMS technical design report, volume II: Physics performance," J. Phys. G 34 (2007) 995.

19. B. C. Allanach et al., "The Snowmass points and slopes: Benchmarks for SUSY searches," in Proc. of the APS/DPF/DPB Summer Study on the Future of Particle Physics (Snowmass 2001) ed. N. Graf, In the Proceedings of APS / DPF / DPB Summer Study on the Future of Particle Physics (Snowmass 2001), Snowmass, Colorado, 30 Jun - 21 Jul 2001, pp P125 arXiv:hep-ph/0202233.

20. J. A. Aguilar-Saavedra et al., "Supersymmetry parameter analysis: SPA convention and project," Eur. Phys. J. C 46 (2006) 43 arXiv:hep-ph/0511344.

21. M. Spiropulu, arXiv:0801.0318 [hep-ex].

22. S. Vahsen [ATLAS Collaboration], presented at the Joint meeting of the Pacific Region Particle Physics Communities, Oct 29 - Nov 3, 2006, Honolulu.

23. T. Yamazaki [ATLAS Collaboration], "Data-driven Estimation of SM Backgrounds for SUSY Searches at the LHC," arXiv:0805.3883 [hep-ex].

24. S. Yamamoto [ATLAS Collaboration], "Strategy for early SUSY searches at ATLAS," arXiv:0710.3953 [hep-ex].

25. G. Arnison et al. [UA1 Collaboration], Phys. Lett. B 147 (1984) 493.

26. G. Arnison et al. [UA1 Collaboration], Phys. Lett. B 139 (1984) 115.

27. See e.g. S. D. Ellis, R. Kleiss and W. J. Stirling, Phys. Lett. B 158 (1985) 341.

28. R. K. Ellis and J. C. Sexton, Nucl. Phys. B 269 (1986) 445.

29. S. D. Ellis, Z. Kunszt and D. E. Soper, Phys. Rev. Lett. 64 (1990) 2121.

30. F. Aversa, M. Greco, P. Chiappetta and J. P. Guillet, Phys. Rev. Lett. 65 (1990) 401

31. S. D. Ellis and D. E. Soper, Phys. Rev. Lett. 74 (1995) 5182 arXiv:hep-ph/9412342.

32. W. T. Giele, E. W. N. Glover and D. A. Kosower, Phys. Rev. Lett. 73 (1994) 2019 arXiv:hep$\mathrm{ph} / 9403347$.

33. S. Frixione, Nucl. Phys. B 507, 295 (1997) arXiv:hep-ph/9706545.

34. S. Frixione, Z. Kunszt and A. Signer, Nucl. Phys. B 467 (1996) 399 arXiv:hep-ph/9512328.

35. W. B. Kilgore and W. T. Giele, Phys. Rev. D 55 (1997) 7183 arXiv:hep-ph/9610433.

36. Z. Nagy, Phys. Rev. D 68 (2003) 094002 arXiv:hep-ph/0307268.

37. P. Nason, S. Dawson and R. K. Ellis, "The Total Cross-Section for the Production of Heavy Quarks in Hadronic Collisions," Nucl. Phys. B 303 (1988) 607.

38. W. Beenakker, H. Kuijf, W. L. van Neerven and J. Smith, "QCD Corrections to Heavy Quark Production in p anti-p Collisions," Phys. Rev. D 40 (1989) 54.

39. M. L. Mangano, P. Nason and G. Ridolfi, "Heavy quark correlations in hadron collisions at nextto-leading order," Nucl. Phys. B 373 (1992) 295.

40. S. Dittmaier, P. Uwer and S. Weinzierl, "NLO QCD corrections to pp - $i$ top anti-top + jet + X," arXiv:0804.4389 [hep-ph].

41. J. Campbell and R. K. Ellis, "Next-to-leading order corrections to $\mathrm{W}+2$ jet and Z +2 jet production at hadron colliders," Phys. Rev. D 65 (2002) 113007.

42. J. Campbell, R. K. Ellis and D. L. Rainwater, "Next-to-leading order QCD predictions for W + 2jet and Z + 2jet production at the CERN LHC," Phys. Rev. D 68 (2003) 094021 arXiv:hep$\mathrm{ph} / 0308195$.

43. J. Campbell, R. K. Ellis, F. Maltoni and S. Willenbrock, "Production of a W boson and two jets with one b-quark tag," Phys. Rev. D 75 (2007) 054015 arXiv:hep-ph/0611348.

44. J. Campbell, R. K. Ellis, F. Maltoni and S. Willenbrock, "Production of a Z boson and two jets with one heavy-quark tag," Phys. Rev. D 73 (2006) 054007 [Erratum-ibid. D 77 (2008) 019903] arXiv:hep-ph/0510362.

45. R. Hamberg, W. L. van Neerven and T. Matsuura, Nucl. Phys. B 359 (1991) 343 [Erratum-ibid. B 644 (2002) 403].

46. R. V. Harlander and W. B. Kilgore, Phys. Rev. Lett. 88 (2002) 201801 arXiv:hep-ph/0201206

47. C. Anastasiou and K. Melnikov, Nucl. Phys. B 646 (2002) 220 arXiv:hep-ph/0207004.

48. C. Anastasiou, L. J. Dixon, K. Melnikov and F. Petriello, Phys. Rev. D 69 (2004) 094008 arXiv:hep-ph/0312266.

49. K. Melnikov and F. Petriello, Phys. Rev. Lett. 96 (2006) 231803. 
50. C. Anastasiou, G. Dissertori and F. Stockli, JHEP 0709 (2007) 018;

51. M. Grazzini, arXiv:0801.3232 [hep-ph].

52. S. Frixione and B. R. Webber, JHEP 0206 (2002) 029 arXiv:hep-ph/0204244.

53. P. Nason, JHEP 0411 (2004) 040 arXiv:hep-ph/0409146.

54. S. Frixione, P. Nason and C. Oleari, JHEP 0711 (2007) 070 arXiv:0709.2092 [hep-ph]].

55. G. Corcella et. al. JHEP 01 (2001) 010.

56. T. Sjöstrand, L. Lönnblad, S. Mrenna and P. Skands arXiv:hep-ph/0308153.

57. S. Frixione and B. R. Webber, arXiv:hep-ph/0612272

58. S. Frixione, P. Nason and B. R. Webber, "Matching NLO QCD and parton showers in heavy flavour production," JHEP 0308, 007 (2003).

59. S. Frixione, P. Nason and G. Ridolfi, "A Positive-Weight Next-to-Leading-Order Monte Carlo for Heavy Flavour Hadroproduction," JHEP 0709, 126 (2007).

60. S. Catani, F. Krauss, R. Kuhn and B. R. Webber, "QCD Matrix Elements + Parton Showers", JHEP 0111 (2001) 063.

61. L. Lonnblad, JHEP 0205 (2002) 046 arXiv:hep-ph/0112284.

62. F. Krauss, JHEP 0208 (2002) 015 arXiv:hep-ph/0205283].

63. M. L. Mangano et al., "Matching matrix elements and shower evolution for top-quark production in hadronic collisions," JHEP 0701, 013 (2007).

64. S. Mrenna and P. Richardson, "Matching matrix elements and parton showers with HERWIG and PYTHIA," JHEP 0405 (2004) 040.

65. J. Alwall et al., "Comparative study of various algorithms for the merging of parton showers and matrix elements in hadronic collisions," Eur. Phys. J. C 53, 473 (2008).

66. M. L. Mangano et al., "ALPGEN, a generator for hard multiparton processes in hadronic collisions," JHEP 0307 (2003) 001.

67. T. Aaltonen et al. [CDF - Run II Collaboration], "Measurement of Inclusive Jet Cross Sections in $Z / \gamma^{*}(\rightarrow e e)+$ jets Production in $p \bar{p}$ Collisions at $\sqrt{s}=1.96 \mathrm{TeV}$," Phys. Rev. Lett. 100 (2008) 102001 arXiv:0711.3717 [hep-ex]].

68. V. M. Abazov et al. [D0 Collaboration], "Measurement of the ratios of the $Z / \gamma^{*}+\geq N$-jet production cross sections to the total inclusive $Z / \gamma^{*}$ cross section in $p \bar{p}$ collisions at $\sqrt{S}=1.96 \mathrm{TeV}$," Phys. Lett. B 658, 112 (2008) arXiv:hep-ex/0608052.

69. T. Aaltonen et al. [CDF Collaboration], "Measurement of the cross section for W-boson production in association with jets in $p \bar{p}$ collisions at $\sqrt{S}=1.96 \mathrm{TeV}, "$ Phys. Rev. D 77, 011108 (2008) arXiv:0711.4044 [hep-ex]].

70. H. Baer, V. Barger and G. Shaughnessy, "SUSY backgrounds to Standard Model calibration processes at the LHC," arXiv:0806.3745 [hep-ph].

71. F. Krauss, A. Schalicke, S. Schumann and G. Soff, "Simulating W / Z + jets production at the Tevatron," Phys. Rev. D 70 (2004) 114009;

72. F. Krauss, A. Schalicke, S. Schumann and G. Soff, "Simulating W / Z + jets production at the CERN LHC," Phys. Rev. D 72 (2005) 054017.

73. N. Lavesson and L. Lönnblad JHEP 07 (2005) 054.

74. F. Maltoni and T. Stelzer JHEP 02 (2003) 027.

75. C. G. Papadopoulos and M. Worek Eur. Phys. J. C50 (2007) 843-856.

76. F. Abe et al. [CDF Collaboration], "Evidence for top quark production in $\bar{p} p$ collisions at $\sqrt{s}=1.8$ TeV," Phys. Rev. D 50 (1994) 2966.

77. F. Abe et al. [CDF Collaboration], "Observation of top quark production in $\bar{p} p$ collisions," Phys. Rev. Lett. 74 (1995) 2626.

78. S. Abachi et al. [D0 Collaboration], "Observation of the top quark," Phys. Rev. Lett. 74 (1995) 2632 .

79. M. Beneke et al., "Top quark physics," arXiv:hep-ph/0003033

80. V. Sorin [CDF and D0 Collaborations], "Tevatron: top quark production and properties," 42nd Rencontres de Moriond on Electroweak Interactions and Unified Theories, La Thuile, Italy, 10-17 Mar 2007, FERMILAB-CONF-07-166-E.

81. M. A. Pleier [CDF and D0 Collaborations], "Top Quark Pair Production and Properties Measurements at the Tevatron," arXiv:0804.4288 [hep-ex].

82. W. M. Yao et al. [Particle Data Group], "Review of particle physics," J. Phys. G 33 (2006) 1, and 2007 partial update for 2008.

83. S. Catani, M. L. Mangano, P. Nason and L. Trentadue, "The Resummation of Soft Gluon in Hadronic Collisions," Nucl. Phys. B 478 (1996) 273 arXiv:hep-ph/9604351. 
84. R. Bonciani et al., "NLL resummation of the heavy-quark hadroproduction cross-section," Nucl. Phys. B 529 (1998) 424;

85. M. Cacciari et al., "The t anti-t cross-section at $1.8-\mathrm{TeV}$ and $1.96-\mathrm{TeV}: \mathrm{A}$ study of the systematics due to parton densities and scale dependence," JHEP 0404 (2004) 068.

86. M. Cacciari, S. Frixione, M. M. Mangano, P. Nason and G. Ridolfi, "Updated predictions for the total production cross sections of top and of heavier quark pairs at the Tevatron and at the LHC," arXiv:0804.2800 [hep-ph].

87. N. Kidonakis and R. Vogt, "Next-to-next-to-leading order soft-gluon corrections in top quark hadroproduction," Phys. Rev. D 68 (2003) 114014 arXiv:hep-ph/0308222.

88. S. Moch and P. Uwer, "Theoretical status and prospects for top-quark pair production at hadron colliders," arXiv:0804.1476 [hep-ph].

89. N. Kidonakis and R. Vogt, "The theoretical top quark cross section at the Tevatron and the LHC," arXiv:0805.3844 [hep-ph].

90. T. Aaltonen et al. [CDF Collaboration], "Measurement of the $p \bar{p} \rightarrow t \bar{t}$ production cross- section and the top quark mass at $\sqrt{s}=1.96-\mathrm{TeV}$ in the all-hadronic decay mode," Phys. Rev. D 76 (2007) 072009 arXiv:0706.3790 [hep-ex]].

91. A. Abulencia et al. [CDF Collaboration], "Measurement of the $t \bar{t}$ Production Cross Section in $p \bar{p}$ collisions at $\sqrt{s}=1.96-\mathrm{TeV}$ using Lepton + Jets Events with Jet Probability $b$-tagging," Phys. Rev. D 74 (2006) 072006 arXiv:hep-ex/0607035.

92. D. E. Acosta et al. [CDF Collaboration], "Measurement of the cross section for $t \bar{t}$ production in $p \bar{p}$ collisions using the kinematics of lepton + jets events," Phys. Rev. D 72 (2005) 052003 arXiv:hep-ex/0504053.

93. D. E. Acosta et al. [CDF Collaboration], "Measurement of the $t \bar{t}$ production cross section in $p \bar{p}$ collisions at $\sqrt{s}=1.96 \mathrm{TeV}$ using dilepton events," Phys. Rev. Lett. 93 (2004) 142001 arXiv:hepex/0404036.

94. V. M. Abazov et al. [D0 Collaboration], "Measurement of the $t \bar{t}$ production cross-section in $p \bar{p}$ collisions using dilepton events," Phys. Rev. D 76 (2007) 052006 arXiv:0706.0458 [hep-ex]].

95. V. M. Abazov et al. [D0 Collaboration], "Measurement of the $t \bar{t}$ production cross section in $p \bar{p}$ collisions at $\sqrt{s}=1.96-\mathrm{TeV}$ using kinematic characteristics of lepton Phys. Rev. D 76 (2007) 092007 arXiv:0705.2788 [hep-ex]].

96. V. M. Abazov et al. [D0 Collaboration], "Measurement of the $p \bar{p} \rightarrow t \bar{t}$ production cross section at $\sqrt{s}=1.96-\mathrm{TeV}$ in the fully hadronic decay channel," Phys. Rev. D 76 (2007) 072007 arXiv:hepex/0612040.

97. V. M. Abazov et al. [D0 Collaboration], "Measurement of the $t$ anti-t production cross section in $\mathrm{p}$ anti-p collisions at $\mathrm{s}^{* *}(1 / 2)=1.96-\mathrm{TeV}$ using secondary vertex b tagging," Phys. Rev. D 74 (2006) 112004 arXiv:hep-ex/0611002.

98. V. M. Abazov et al. [D0 Collaboration], "Measurement of the $t$ anti-t production cross section in $\mathrm{p}$ anti-p collisions at $\mathrm{s}^{* *}(1 / 2)=1.96-\mathrm{TeV}$ using lepton + jets events with lifetime b-tagging," Phys. Lett. B 626 (2005) 35 arXiv:hep-ex/0504058.

99. J. Pumplin, D. R. Stump, J. Huston, H. L. Lai, P. Nadolsky and W. K. Tung, "New generation of parton distributions with uncertainties from global QCD analysis," JHEP 0207 (2002) 012 arXiv:hep-ph/0201195.

100. A. D. Martin, R. G. Roberts, W. J. Stirling and R. S. Thorne, "MRST2001: Partons and alpha(s) from precise deep inelastic scattering and Tevatron jet data," Eur. Phys. J. C 23 (2002) 73 arXiv:hep-ph/0110215.

101. M. Czakon, A. Mitov and S. Moch, "Heavy-quark production in massless quark scattering at two loops in QCD," Phys. Lett. B 651 (2007) 147 arXiv:0705.1975 [hep-ph]].

102. M. Czakon, A. Mitov and S. Moch, "Heavy-quark production in gluon fusion at two loops in QCD," Nucl. Phys. B 798 (2008) 210 arXiv:0707.4139 [hep-ph]].

103. M. Czakon, "Tops from Light Quarks: Full Mass Dependence at Two-Loops inQCD," arXiv:0803.1400 [hep-ph].

104. J. G. Korner, Z. Merebashvili and M. Rogal, "One-loop amplitudes for four-point functions with two external massive quarks and two external massless partons up to $\mathrm{O}\left(\epsilon^{2}\right)$," Phys. Rev. D 73 (2006) 034030 arXiv:hep-ph/0511264.

105. J. G. Korner, Z. Merebashvili and M. Rogal, "NNLO $\mathcal{O}\left(\alpha_{s}^{4}\right)$ results for heavy quark pair production in quark-antiquark collisions: The one-loop squared contributions," arXiv:0802.0106 [hep-ph].

106. F. Abe et al. [CDF Collaboration], "Further Properties of High-Mass Multijet Events at the Fermilab Proton-Antiproton Collider," Phys. Rev. D 54 (1996) 4221 arXiv:hep-ex/9605004.

107. A. Bhatti et al., "Determination of the jet energy scale at the Collider Detector at Fermilab," Nucl. Instrum. Meth. A 566 (2006) 375 arXiv:hep-ex/0510047. 